\title{
TIME DEPENDENT NONEQUILIBRIUM IONIZATION OF TRANSITION REGION LINES OBSERVED WITH IRIS
}

\author{
Juan Martínez-Sykora ${ }^{1,2}$, Bart De Pontieu ${ }^{1,3}$, Viggo H. Hansteen ${ }^{1,3}$, and Boris Gudiksen ${ }^{3}$ \\ ${ }^{1}$ Lockheed Martin Solar and Astrophysics Laboratory, Palo Alto, CA 94304, USA; j.m.sykora@astro.uio.no \\ 2 Bay Area Environmental Research Institute, Petaluma, CA, USA \\ ${ }^{3}$ Institute of Theoretical Astrophysics, University of Oslo, P.O. Box 1029 Blindern, NO-0315 Oslo, Norway \\ Received 2015 September 28; accepted 2015 November 30; published 2016 January 20
}

\begin{abstract}
The properties of nonstatistical equilibrium ionization of silicon and oxygen ions are analyzed in this work. We focus on five solar targets (quiet Sun; coronal hole; plage; quiescent active region, AR; and flaring AR) as observed with the Interface Region Imaging Spectrograph (IRIS). IRIS is best suited for this work owing to the high cadence (up to $0.5 \mathrm{~s}$ ), high spatial resolution (up to 0 "' 32 ), and high signal-to-noise ratios for O IV $\lambda 1401$ and $\mathrm{Si}$ Iv $\lambda 1402$. We find that the observed intensity ratio between lines of three times ionized silicon and oxygen ions depends on their total intensity and that this correlation varies depending on the region observed (quiet Sun, coronal holes, plage, or active regions) and on the specific observational objects present (spicules, dynamic loops, jets, microflares, or umbra). In order to interpret the observations, we compare them with synthetic profiles taken from 2D self-consistent radiative MHD simulations of the solar atmosphere, where the statistical equilibrium or nonequilibrium treatment of silicon and oxygen is applied. These synthetic observations show vaguely similar correlations to those in the observations, i.e., between the intensity ratios and their intensities, but only in the nonequilibrium case do we find that (some of) the observations can be reproduced. We conclude that these lines are formed out of statistical equilibrium. We use our time-dependent nonequilibrium ionization simulations to describe the physical mechanisms behind these observed properties.
\end{abstract}

Key words: line: profiles - shock waves - Sun: atmosphere - Sun: chromosphere - Sun: transition region

Supporting material: animations

\section{INTRODUCTION}

The solar chromosphere and transition layer contain a large number of physical transitions. The ratio of magnetic to hydrodynamic forces changes, the plasma goes from being optically thick to optically thin, thermal conduction becomes drastically more efficient, and the ionization state of hydrogen and helium changes sufficiently to have an impact on the energy balance. These transitions make it difficult to translate observations into physical models of the atmosphere, but likewise equally difficult to model, as a large number of physical forces and effects need to be included in order for a model to be successful. Models should be able to teach us what the observations mean, but in the chromosphere and transition region, some of the most intense spectral lines we observe are formed by ions out of ionization equilibrium, which has only recently been possible to include in multidimensional numerical simulations (Olluri et al. 2015).

In this work we will focus on the emission lines O IV $\lambda 1401$ and $\operatorname{Si}$ IV $\lambda 1402$, which are formed in the lower transition region. Both lines are readily observed with the Interface Region Imaging Spectrograph (IRIS; De Pontieu et al. 2014) at high spatial resolution, temporal cadence, and signal-to-noise ratio. The observed ratio between the intensities of these lines differs considerably from that derived from numerical models assuming statistical equilibrium (SE) ionization. Olluri et al. (2015) show that these discrepancies are reduced when using nonequilibrium ionization of oxygen and silicon and oxygen abundances from Asplund et al. (2009) and "coronal" abundances for silicon (Feldman 1992).

Nonequilibrium ionization becomes important when the timescales of ionization and recombination are longer than the dynamic timescales characterizing the atmosphere. In the solar atmosphere this is likely to be the case in the upper chromosphere, transition region, and corona. This is important not only for understanding the diagnostic signatures of spectral lines formed in these regions but also for the thermodynamic properties of the atmosphere. For example, in the upper chromosphere and in the transition region the nonequilibrium of the hydrogen and helium ionization state will impact the energetics of the plasma (Leenaarts et al. 2007; Golding et al. 2014).

The interpretation of observed emission lines will differ depending on whether or not nonequilibrium ionization is taken into account. This is revealed in the following short list of theoretical studies in which the results of nonequilibrium ionization are compared with ions formed assuming SE and found to be important: evaporation flows produced by nanoflare heating in 1D hydro models (Bradshaw \& Cargill 2006); small-scale impulsive heating produces nonequilibrium ionization (Bradshaw \& Klimchuk 2011). Upwardly propagating shocks in the chromosphere and transition region will most likely give rise to nonequilibrium ionization in several lines (Judge et al. 1997). Recently, it has also been shown that transition region UV lines can depart from equilibrium ionization not only in 1D models (Joselyn et al. 1979; Hansteen 1993, among others) but also in 3D MHD models (Olluri et al. 2013a, 2013b).

Thus, assuming SE in cases where nonequilibrium ionization is important when analyzing observations will likely lead to erroneous conclusions as to the state of the atmosphere. The difficulty is, of course, how to take these nonequilibrium effects into account. Bradshaw \& Klimchuk (2011) are only able to reproduce observed differential emission measures (DEMs) of 
Table 1

Description of the Observations

\begin{tabular}{|c|c|c|c|c|c|c|c|}
\hline Name & Time & pos & \# Steps & FOV & $\mathrm{Cad}$ & Exp & Other \\
\hline QS1 & 2013 Nov 27 07:15:57 UT & $-46^{\prime \prime}, 381^{\prime \prime}$ & 64 & $127^{\prime \prime} \times 175^{\prime \prime}$ & $1971 \mathrm{~s}$ & $32 \mathrm{~s}$ & Quiet Sun with filament \\
\hline $\mathrm{CH} 1$ & 2015 Mar 02 18:04:33 UT & $507^{\prime \prime},-551^{\prime \prime}$ & 400 & $141^{\prime \prime} \times 175^{\prime \prime}$ & $12526 \mathrm{~s}$ & $32 \mathrm{~s}$ & Coronal hole close to the limb \\
\hline P11 & 2014 Apr 04 05:16:20 UT & $113^{\prime \prime}, 368^{\prime \prime}$ & 96 & $33^{\prime \prime} \times 174^{\prime \prime}$ & $3041 \mathrm{~s}$ & $32 \mathrm{~s}$ & Plage without sunspots \\
\hline AR1 & 2013 Nov 23 15:36:09 UT & $-39^{\prime \prime}, 226^{\prime \prime}$ & 64 & $127^{\prime \prime} \times 175^{\prime \prime}$ & $1973 \mathrm{~s}$ & $32 \mathrm{~s}$ & AR without flaring or emergence \\
\hline AR2 & 2014 Aug 15 22:36:09 UT & $-306^{\prime \prime}, 115^{\prime \prime}$ & 400 & $141^{\prime \prime} \times 174^{\prime \prime}$ & $12774 \mathrm{~s}$ & $32 \mathrm{~s}$ & AR with some emergence \\
\hline
\end{tabular}

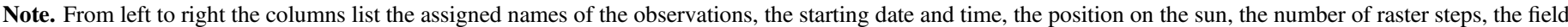
of view of the raster, the raster cadence, exposure time, and a brief description of the target.

impulsive heating and cooling loops when nonequilibrium ionization is included. Assuming SE instead of nonequilibrium ionization when analyzing density-dependent emission line ratios gives derived densities that differ by an order of magnitude (Olluri et al. 2013b). Similarly, assuming SE when searching for the effects of $\kappa$-distributions, i.e., non-Gaussian profiles, on line emission (Dudík et al. 2014) is problematic since nonequilibrium ionization will change the intensity ratios between lines. One must therefore be careful when comparing O IV $\lambda 1401$ and Si IV $\lambda 1402$ lines for two main reasons: (1) the high likelihood of nonequilibrium ionization, and (2) the peak formation temperatures of these lines are not exactly the same.

This paper combines IRIS observations with 2D radiative MHD simulations that include nonequilibrium ionization of both silicon and oxygen (Olluri et al. 2015), using the Bifrost code (Gudiksen et al. 2011). First, we describe the IRIS data processing and the setup of the selected IRIS data (Section 2). A description of the simulations and how the synthetic data are calculated is given in Section 3. We give an overview of the observations in Section 4.1 and compare these with the synthetic observations using SE in Section 4.2.1. Finally, we present an analysis of synthetic observations using nonequilibrium ionization in Section 4.2.2. In Section 5 we finish the paper with a discussion and conclusions.

\section{OBSERVATIONS}

IRIS obtains spectra in passbands from 1332 to $1358 \AA$ with spectral pixel size of $12.98 \mathrm{~m} \AA, 1389-1407 \AA$ with spectral pixel size of $12.72 \mathrm{~m} \AA$, and $2783-2834 \AA$ with spectral pixel size of $25.46 \mathrm{m \AA}$. These passbands include bright spectral lines formed in the chromosphere, e.g., $\quad \mathrm{Mg}_{\text {II }} h \lambda 2803$ and Mg II $k \lambda 2796$, in the upper chromosphere/lower transition region, e.g., $\mathrm{C}_{\text {II }} \lambda 1334 / \lambda 1335$, and in the transition region, e.g., Si IV $\lambda 1394 / \lambda 1403$. Spectral rasters sample spatial regions with sizes of up to $130^{\prime \prime} \times 175^{\prime \prime}$ at a variety of spatial samplings (from 0". 166 and up). In addition, IRIS can take slitjaw images (SJIs) with different filters that have spectral windows dominated by emission from these spectral lines with a spatial resolution of 0 ". 33 and up. SJI 2796 is centered on Mg II $k$ at $2796 \AA$ and has a $4 \AA$ bandpass, SJI 2830 is centered on the $\mathrm{Mg}$ II $h$ wing and has a $4 \AA$ bandpass, SJI 1330 is centered at $1340 \AA$ and has a $55 \AA$ bandpass, and SJI 1400 is centered at $1390 \AA$ and has a $55 \AA$ bandpass. For more information on IRIS, we refer the reader to De Pontieu et al. (2014).

We focus on the spectral lines O IV $\lambda \lambda 1399,1401$, and 1404 and Si IV $\lambda 1402$. Inspection of SJI images allows us to identify various typical solar features. We have selected ondisk targets, i.e., quiet Sun (QS), coronal hole $(\mathrm{CH})$, plage $(\mathrm{Pl})$, and active region (AR) as listed in Table 1 . The selected observations have in common that all of them are spatial rasters (as opposed to sit-and-stare observations) and all have an exposure time of $32 \mathrm{~s}$, ensuring a good signal-to-noise ratio. We use level 2 data that have been calibrated for dark current and include flat-field and geometrical correction (De Pontieu et al. 2014). Other properties of the observations are listed in Table 1.

\section{SIMULATIONS}

We performed a 2D radiative MHD simulation including thermal conduction along the magnetic field lines using the Bifrost code (see Gudiksen et al. 2011, for details). The radiative transfer in the photosphere and lower chromosphere is solved using the method developed by Nordlund (1982) and the inclusion of scattering by Skartlien et al. (2000). We refer to Hayek et al. (2010) for details of this implementation in the Bifrost code. In the chromosphere and transition region, the non-LTE radiative losses follow Carlsson \& Leenaarts (2012) recipes. For the corona, we assume optically thin radiative losses. In addition, oxygen and silicon ionization has been calculated using time-dependent non-SE ionization for synthetic observational purposes (Olluri et al. 2013a).

The 2D simulation spans the region from the upper convection zone (2.5 Mm below the photosphere) to the lower corona (14 Mm above the photosphere) and $16 \mathrm{Mm}$ horizontally. This domain is resolved with $512 \times 496$ grid points where the grid cell size along the horizontal axis is uniform $(\sim 31 \mathrm{~km})$ and along the vertical axis it is nonuniform. The latter allows us to have a smaller grid size in places where it is needed, such as in the photosphere and chromosphere $(\sim 25 \mathrm{~km})$, while the grid spacing expands in the convection zone and in the corona. The initial magnetic field configuration is vertical and uniform, with a mean unsigned magnetic field strength of $5 \mathrm{G}$. The horizontal boundary conditions are periodic. The bottom boundary is open, with the entropy fixed at a value that allows the simulation to have an effective temperature similar to the Sun. The top boundary allows waves to propagate through it.

This setup leads to a simulation that is dominated by magnetoacoustic shocks that propagate along the magnetic field into the corona, pushing the transition region upward temporarily as they do so. This behavior is similar to that seen in type I spicules and/or dynamic fibrils and has been described by several authors (e.g., Hansteen et al. 2006; De Pontieu 2007a; Heggland et al. 2007; Martínez-Sykora et al. $2009 b$ ). While the presence of type I spicules fits well with observed phenomena on the Sun, several other phenomena are noticeable mainly by their absence. The model lacks type II spicules (e.g., De Pontieu et al. 2007b; Martínez-Sykora et al. 2011b; Goodman 2012; Martínez-Sykora et al. 2013), 


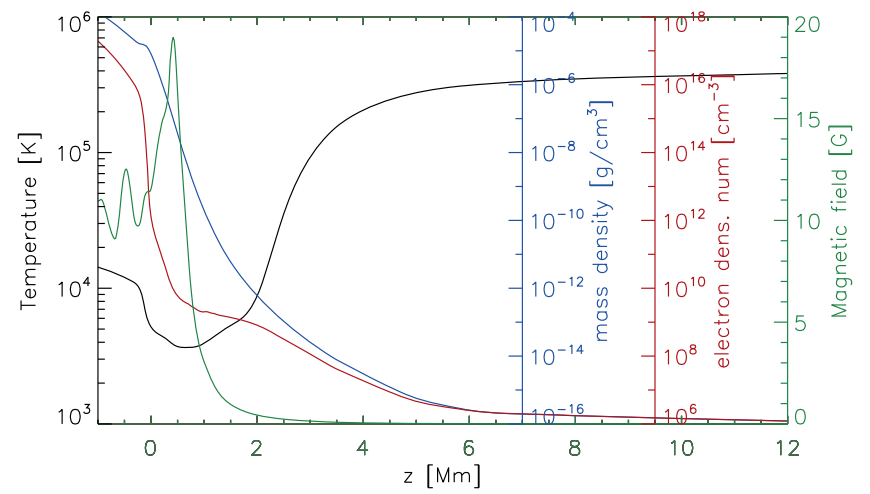

Figure 1. Horizontal and time averages for the temperature (black line), unsigned magnetic field (green), mass density (blue), and electron density number (red) as a function of height.

"unresolved fine structure" (Hansteen et al. 2014), flux emergence (e.g., Martínez-Sykora et al. 2008, 2009a; TortosaAndreu \& Moreno-Insertis 2009; Fang et al. 2012), and/or more violent eruptions (Archontis \& Hansteen 2014). This is in part because of the choice of an extremely simple magnetic geometry that precludes phenomena dependent on complex field geometries such as microflares or unresolved fine structure, but may also be due to insufficient spatial resolution and/or missing physics such as partial ionization effects that are not included in the described runs. In any case, the interpretation of the observables detailed in this paper, while giving insight into the processes involved, is therefore necessarily limited to the structures and dynamics that the simulation is reproducing.

We run the simulation for roughly an hour solar time until it settles down and any transients from the initial conditions are gone. The corona is self-maintained with temperatures up to $6.5 \times 10^{5} \mathrm{~K}$ and average values of $4 \times 10^{5} \mathrm{~K}$ (Figure 1 ) produced by the electrical current (including from magnetic reconnection) dissipated in the corona as a result of footpoints braiding in the photosphere (Gudiksen \& Nordlund 2005; Hansteen et al. 2010; Gudiksen et al. 2011; Martínez-Sykora et al. 2011a; Hansteen et al. 2015). These currents are a consequence of the convective motions in the photosphere and dissipate effectively when magnetic field gradients become large (see the references cited above). Once we reach SE in the simulation, we turn on the time-dependent nonequilibrium ionization of oxygen and silicon, let it run until transients are gone, and continue for another 40 minutes solar time. Figure 1 shows the horizontal and time (15 minutes of time integration) averages for the temperature, unsigned magnetic field, mass density, and electron density as a function of height. Around the transition region, the electron density number is $\sim 10^{9} \mathrm{~cm}^{-3}$.

\subsection{Synthetic Observations}

We synthesized from the simulation the emission of O IV $\lambda \lambda 1393,1401$, and 1404 and Si IV $\lambda \lambda 1402$ and 1393 assuming the optically thin approximation. We perform this calculation in two manners: (1) Assuming statistical thermal equilibrium (SE) using CHIANTI v.7.0 (Dere et al. 2009; Dere 2011) with the ionization balance chianti.ioneq, available in the CHIANTI distribution, i.e., following the same prescription as Hansteen et al. (2010) and Martínez-Sykora et al. (2011a):

$$
I(\nu)=\int_{l} \phi(\nu) A_{b} n_{\mathrm{e}} n_{\mathrm{H}} G\left(T, n_{\mathrm{e}}\right) d l,
$$

where $l$ is length along the line of sight (LOS). $A_{b}, n_{\mathrm{e}}, n_{\mathrm{H}}$, and $G\left(T, n_{\mathrm{e}}\right)$ represent the abundance of the emitting element, the electron and the hydrogen densities, and the contribution function, respectively. The electron density is taken from the equation-of-state lookup table of the simulation. We create a lookup table of the contribution function $\left(G\left(T, n_{\mathrm{e}}\right)\right)$ using the Solarsoft package for IDL ch_synthetic.pro, where the keyword GOFT is selected. The line profile is computed assuming Doppler broadening:

$$
\phi_{\nu}=\frac{1}{\pi^{1 / 2} \Delta \nu_{D}} \exp \left[-\left(\frac{\Delta \nu-\nu \boldsymbol{u} \cdot \boldsymbol{n} / c}{\Delta \nu_{D}}\right)^{2}\right],
$$

where $\Delta \nu=\nu-\nu_{o}$ is the frequency difference from the rest frequency of the line, $\boldsymbol{u}$ and $\boldsymbol{n}$ are the velocity and the unit vector along the LOS, respectively, and $c$ is the speed of light. The thermal broadening profile corresponds to a width:

$$
\Delta \nu_{D}=\frac{\nu_{o}}{c} \sqrt{\frac{2 k T}{m_{A}}}
$$

where $m_{A}$ is the mass of the radiating ion and $k$ is the Boltzmann constant.

(2) Taking into account the non-SE of silicon and oxygen (Olluri et al. 2013a, 2015) and using the upper-level population directly:

$$
I(\nu)=\frac{h \nu}{4 \pi} \int_{l} \phi(\nu) A_{u l} n_{\mathrm{u}} d l,
$$

where $h \nu_{o}$ is the energy of the transition. $A_{u l}$ and $n_{\mathrm{u}}$ are the Einstein constant decay rate and the population density of the upper level of the transition. We synthesized observations for two conditions: (1) assuming photospheric abundances (Grevesse \& Sauval 1998) for both elements, i.e., oxygen and silicon; (2) for comparison, also using the prescription of abundances suggested by Olluri et al. (2015), i.e., Asplund et al. (2009) photospheric abundances for oxygen and coronal abundances for silicon (Feldman 1992).

\section{RESULTS}

\subsection{Comparison of $\mathrm{O} I V_{\text {IV }}$ and Si IV Using IRIS Observations}

In this paper we focus on the O IV $1401 \AA$ and Si IV $1402 \AA$ profiles, for this we use IRIS observations for various targets on the Sun: quiet Sun, coronal hole, plage, and two active regions, as listed in Table 1.

\subsubsection{QS and CH IRIS Observations}

For the first quiet-Sun target (QS1), Figure 2 shows raster maps of the intensity ratio of Si IV $\lambda 1402$ to O IV $\lambda 1401$ (panel (A)), Si Iv intensity (panel (C)), relative Doppler shift (panel (E)) and line width raster maps (panel $(\mathrm{G})$ ), and O IV intensity (panel (D)), relative Doppler shift (panel (F)), and line width raster maps (panel $(\mathrm{H})$ ), and SJI 1400 $\AA$ map at $t=7: 33: 09$ UT (panel (B)). The corresponding movie of the SJI 1400 (Movie 1) is in the online material. The intensity has been integrated over a spectral range that is wide enough to include 


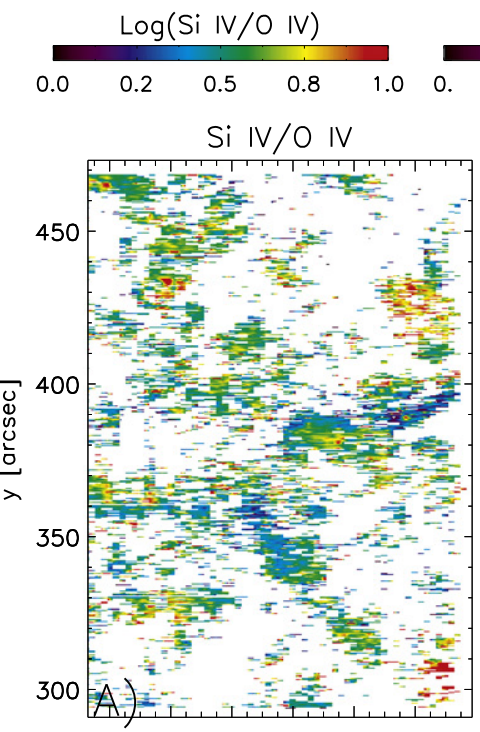

SJI 1400

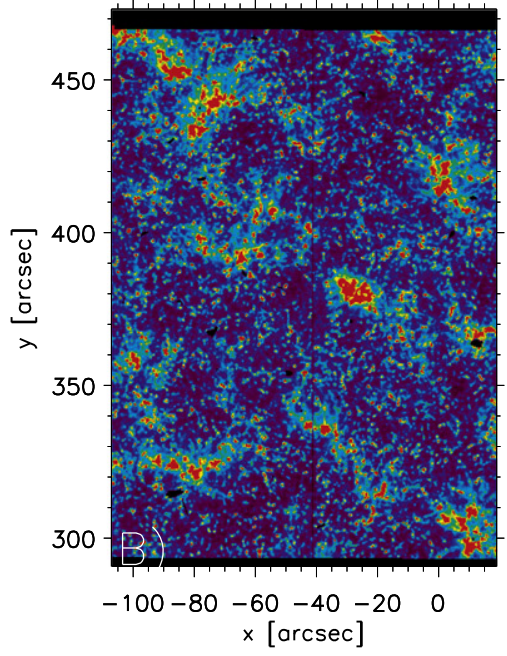

$\operatorname{lnt}[\mathrm{DN}]$

520. 1041. 1561. 2082.

Si IV Int

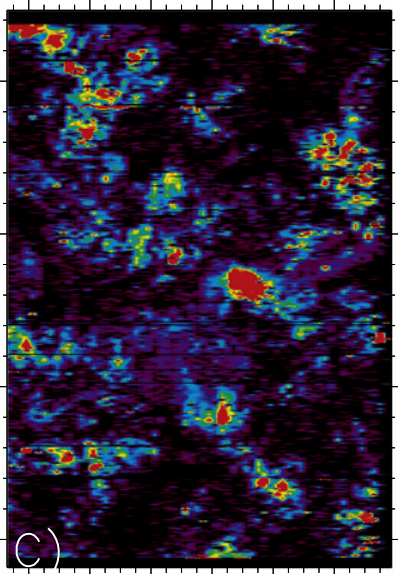

O IV Int

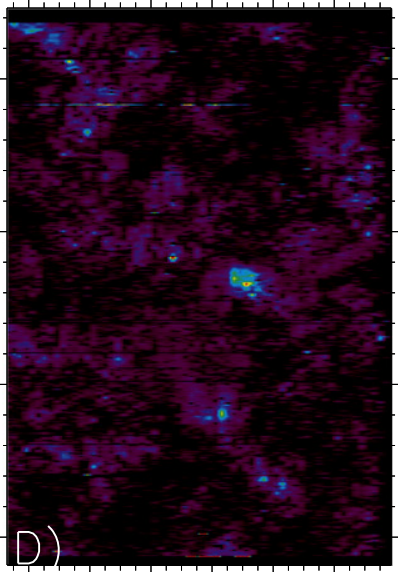

$-100-80-60-40-200$

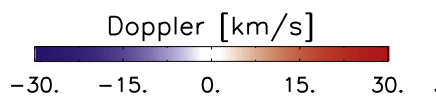

Si IV Doppler

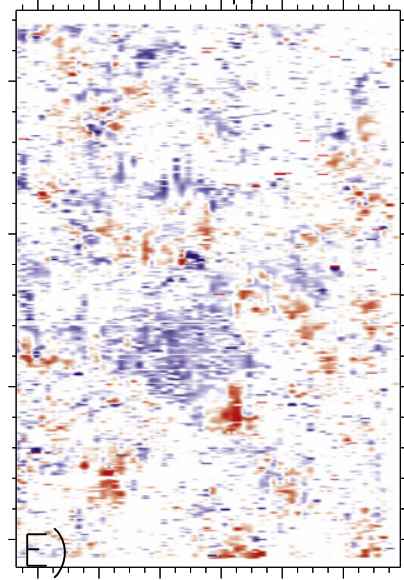

O IV Doppler

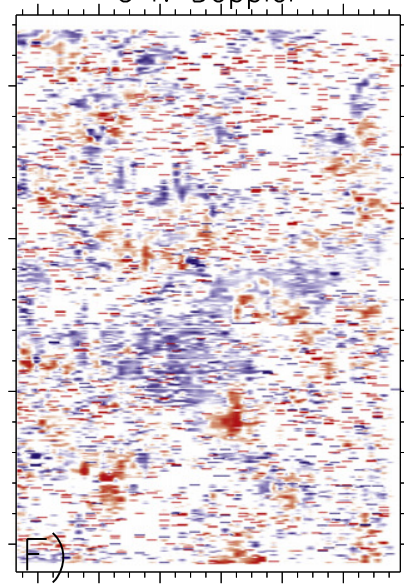

$-100-80-60-40-200$

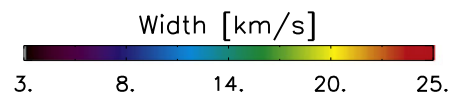

Si IV Width

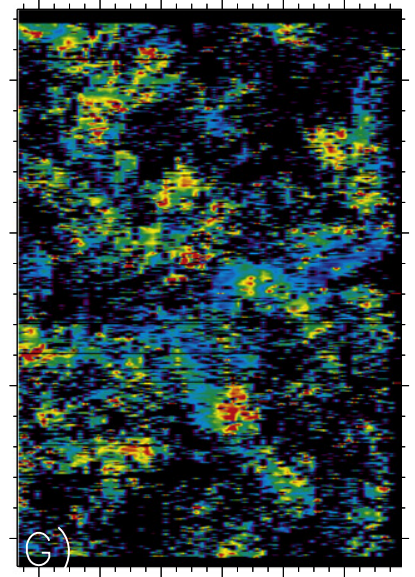

O IV Width

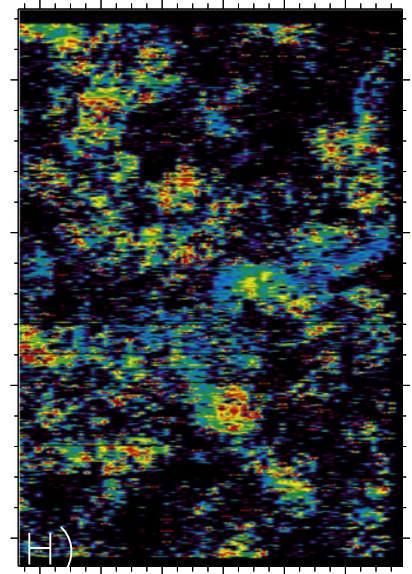

$-100-80-60-40-200$
$\times[\operatorname{arcsec}]$

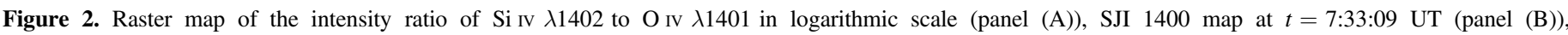

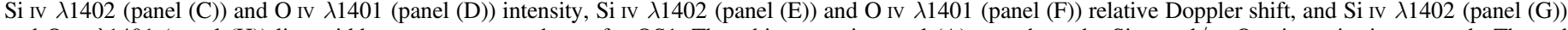

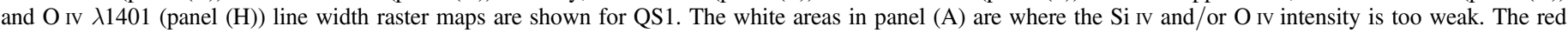
(blue), i.e., positive (negative) relative Doppler shift is downward (upward) velocity (see the corresponding SJI 1400 Movie 1).

(Animations ( $a$ and $b$ ) of this figure are available.)

emission from the entire spectral line without adding contamination from other spectral lines or continuum. The relative Doppler shift has been calculated using a single Gaussian fit of the peak of the profile in order to reduce the effects of contributions from other components in multicomponent profiles. The line width has been calculated using a single Gaussian fit of the full profile in order to take into account, to some degree, the multicomponent contribution. Note that the intensity ratio (panel (A)) is displayed using a logarithmic scale.

Most of the Si IV $\lambda 1402$ and O IV $\lambda 1401$ emission in the quiet-Sun region (QS1) and in the coronal hole region $(\mathrm{CH} 1$, not shown here, although the Movie 2 of the SJI 1400 is in the online material) is concentrated in the network (Sivaraman \& Livingston 1982; Sivaraman et al. 2000), and the emission in the internetwork is almost negligible (panels (C) and (D) of Figure 2). The enhanced network is mainly dominated by redward Doppler shifts (panels $(\mathrm{E})$ and $(\mathrm{F})$ ). Some features reveal elongated shapes such as the one around $\left[-20^{\prime \prime}, 470^{\prime \prime}\right]$.
Note that the SJI $1400 \AA$ map (panel (B)) shows small grain structures everywhere (even in the internetwork) that have almost no emission or are very faint in Si IV and O IV (Martínez-Sykora et al. 2015b). These grains have been explained as chromospheric acoustic shocks (Carlsson \& Stein 1992, 1997; Carlsson et al. 1997; Judge et al. 1997; Steffens et al. 1997; Wedemeyer et al. 2004). The faint emission in these transition region lines of these grains shows $\mathrm{red} /$ blue grain structure in the Doppler shift raster maps, which is a combination of the shock pattern going through the transition region and noise.

We find that the intensity, Doppler shift, and line width maps for both lines are very similar. Presumably, this implies that we are looking at the same features and the intensity ratio is an observational property of this observed feature. This similarity is also found in the other selected regions; see Figures 3-5.

Returning to Figure 2, one can appreciate that many regions with some activity and large $\mathrm{Si}$ IV emission (see top right side or 


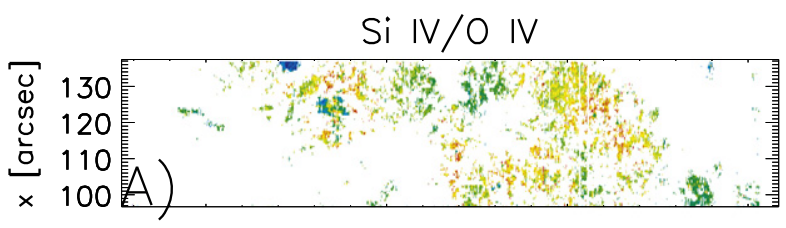

Si IV Int
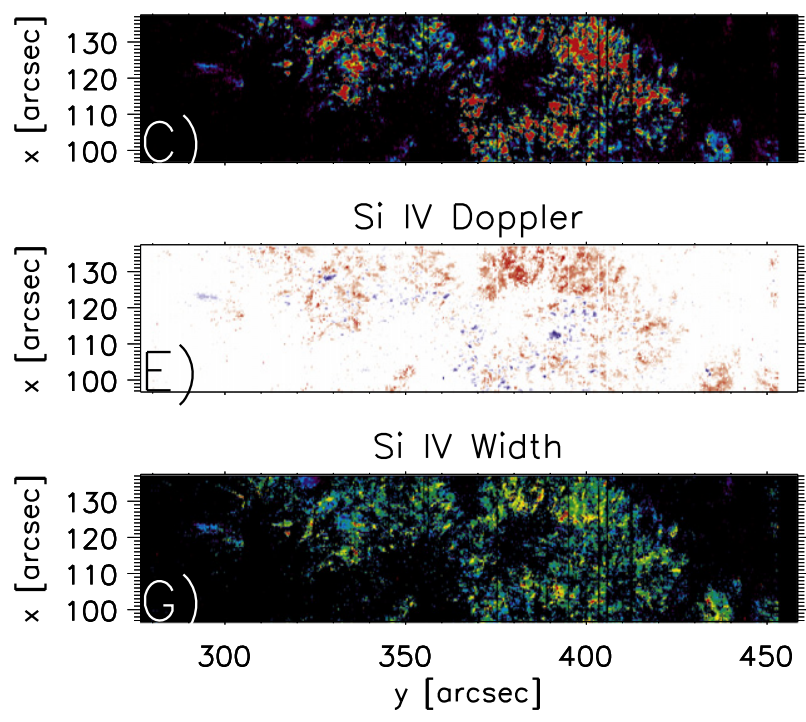
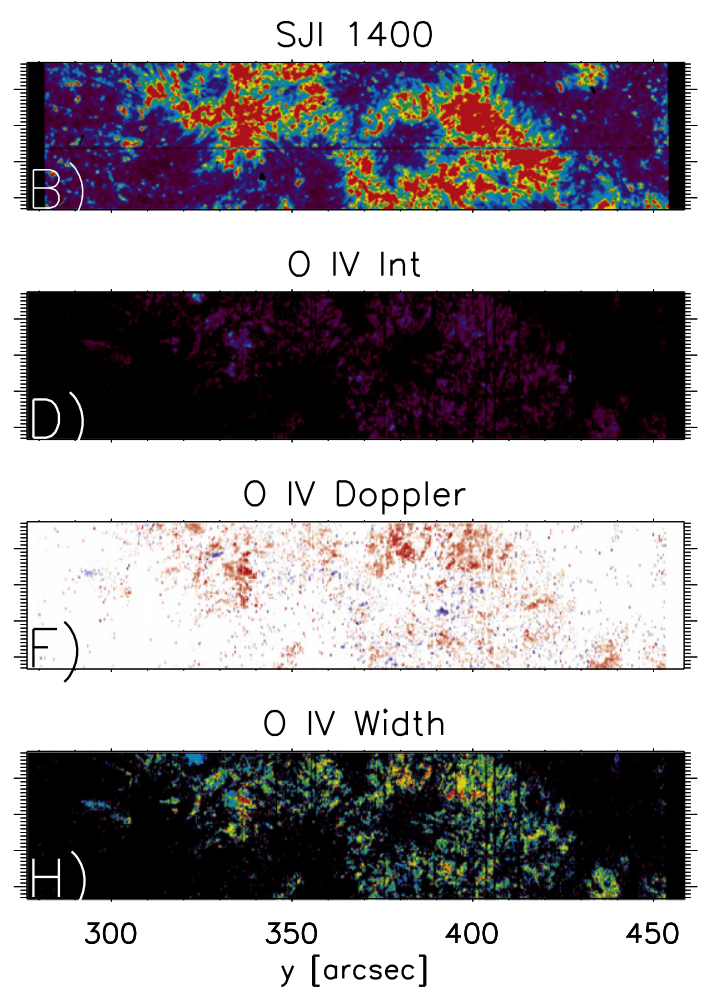

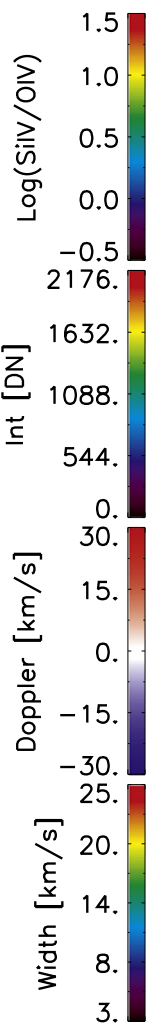

Figure 3. Same layout as Figure 2 for the plage region (P11). The SJI 1400 map is at $t=05: 42: 29$ UT. The $x$-and $y$-axes have been flipped for aesthetic reasons (see SJI 1400 Movie 3).

(An animation of this figure is available.)

lower right side of the raster maps) produce large intensity ratios. In fact, these maps suggest that the intensity ratio increases with Si IV intensity. However, there are exceptions, such as in the bright grains around [-40", 370"] and around $\left[-40^{\prime \prime}, 340^{\prime \prime}\right]$ : both have rather low intensity ratios. These two bright points have the largest $\mathrm{Si}$ IV intensities in the FOV. Therefore, the strongest bright points in Si IV in the raster do not have the largest intensity ratios. The coronal hole rasters (CH1) show similar behavior to that in QS1 in that the intensity ratio increases with $\mathrm{Si}$ IV intensity.

\subsubsection{Plage IRIS Observations}

In the plage region (P11, Figure 3 and Movie 3), the Si IV and O IV intensity raster maps show elongated fibril structures emerging from the concentrations of the magnetic field. These structures have a preferential red Doppler shift. In the plage region, again one can appreciate that the intensity ratio of Si IV $\lambda 1402$ to O IV $\lambda 1401$ increases with intensity. Note the exception around $\left[125^{\prime \prime}, 340^{\prime \prime}\right]$. This corresponds to a spot where the SJI 1400 and SJI 1330 intensity fades a bit compared to the surroundings (not appreciable in the figure owing to the color saturation). This shares several similarities in the chromosphere with a pore, though we do not find any pore in the photosphere below. In addition, in the raster map FOV there is a small pore around $[x, y]=\left[134^{\prime \prime}, 320^{\prime \prime}\right]$ with low intensity in Si IV and $\mathrm{O}$ IV and narrow $\mathrm{Si}$ IV and $\mathrm{O}$ IV profiles. There, the intensity ratio and line widths are very low.

\subsubsection{IRIS Observations of a Quiescent AR}

The active region (AR1) shown in Figure 4 and Movie 4 is quiescent in the sense that we do not see dynamic or energetic phenomena such as flares, flux emergence, or very high velocities. The region features a sunspot surrounded by a plage. Inside the sunspot, the Si IV and O IV intensity is rather faint, with a mixture of regions with up-and-down flow seen in the Doppler shift raster maps. We also see narrow profiles similar to the pore in P11. The penumbra has an enhanced brightening in the Si IV and O IV intensity, revealing fibril structures with large red Doppler shifts and broad profiles. The enhanced network and plage also show strong intensities with large redshifts and broad profiles. In addition to this, one can appreciate loops in the Si IV and O IV intensities with rather low emission and, in many cases, with bidirectional flows connecting the sunspot with the plage. The raster maps in Figure 4 reveal that the stronger the $\mathrm{Si}$ Iv intensity, the higher is the intensity ratio of $\mathrm{Si}$ IV $\lambda 1402$ and O IV $\lambda 1401$. However, the center of the penumbra, which has stronger emission in Si IV than the enhanced network, does not reveal as high intensity ratios of Si IV to $\mathrm{O}$ IV as in the enhanced network. In addition, as mentioned before for the pore in the Pl1 region, the umbra shows a very low intensity ratio. It is also interesting to appreciate that the small mottles or dynamic fibrils around $[x, y]=\left[0^{\prime \prime},-220^{\prime \prime}\right]$ (on the right-hand side of the umbra next to the penumbra) have some large O IV widths, while Si IV widths are smaller. This indicates that the processes driving nonthermal broadening act differently on these two lines. 

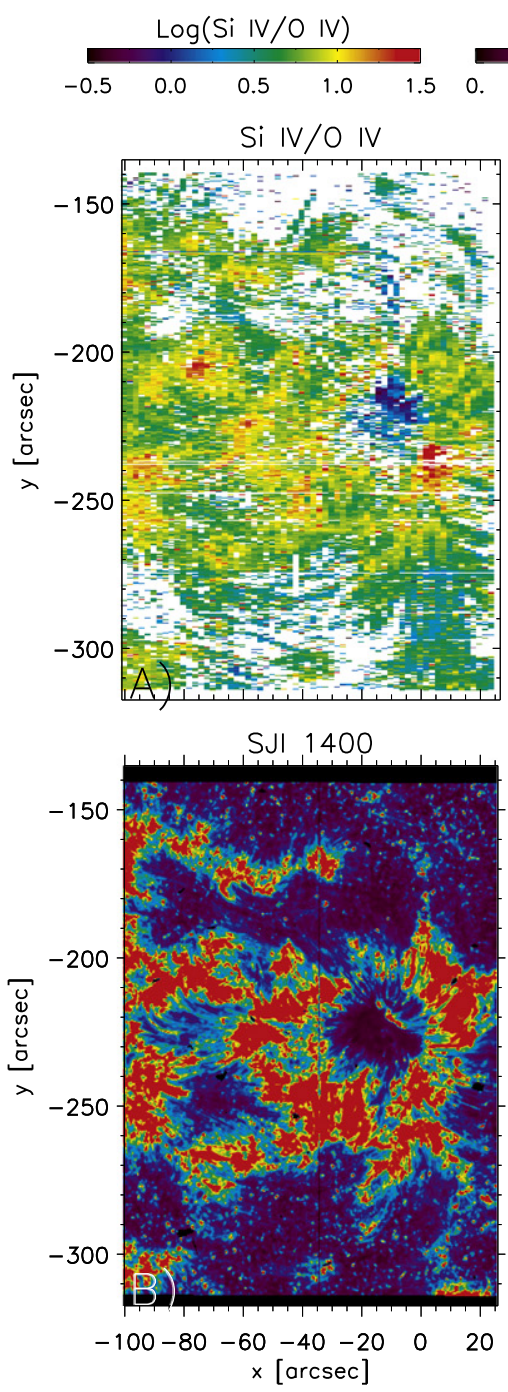
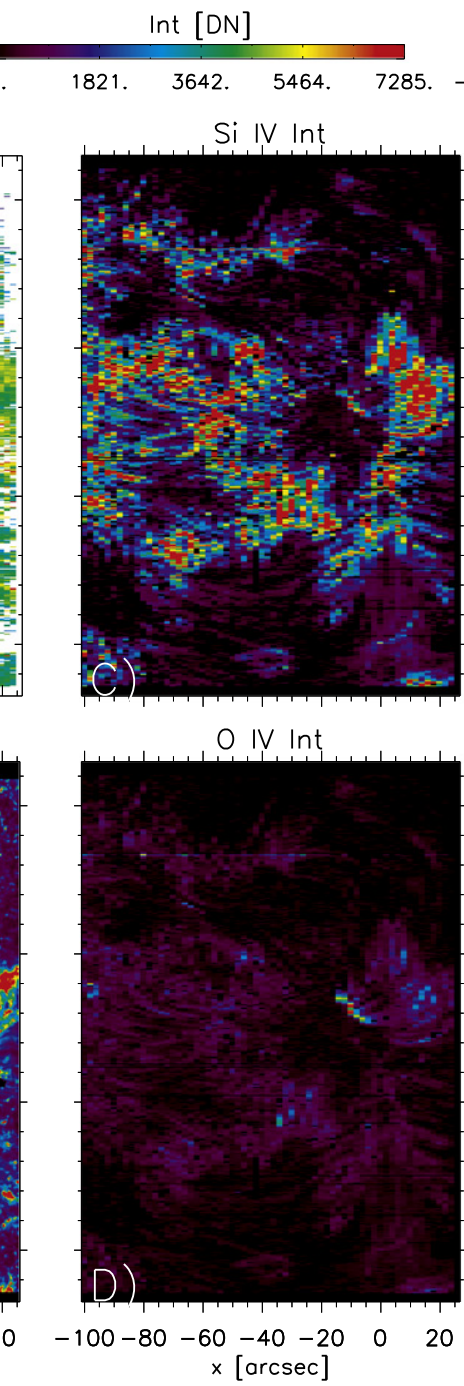
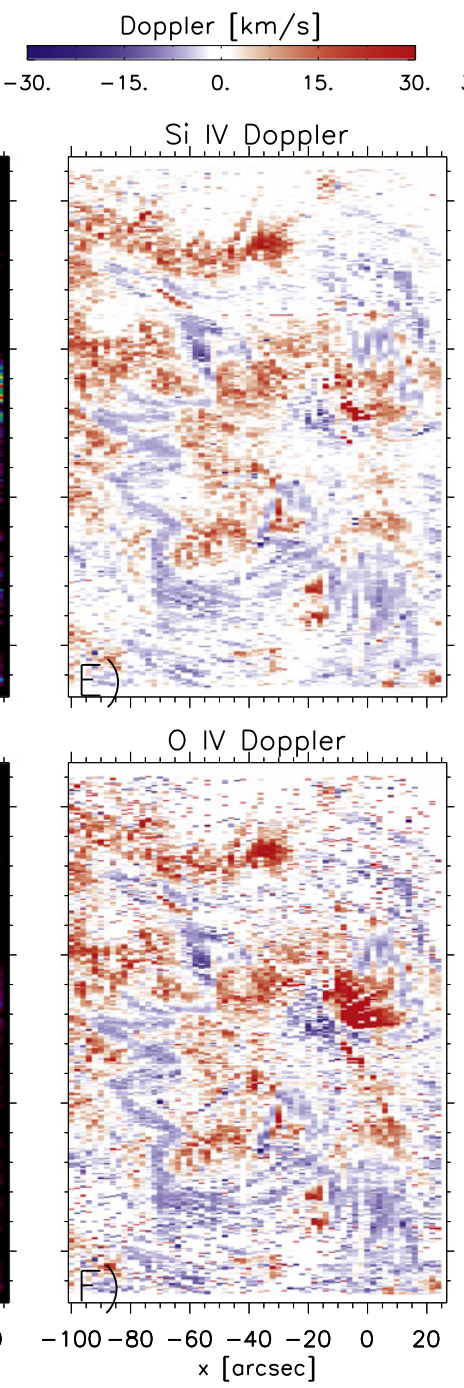
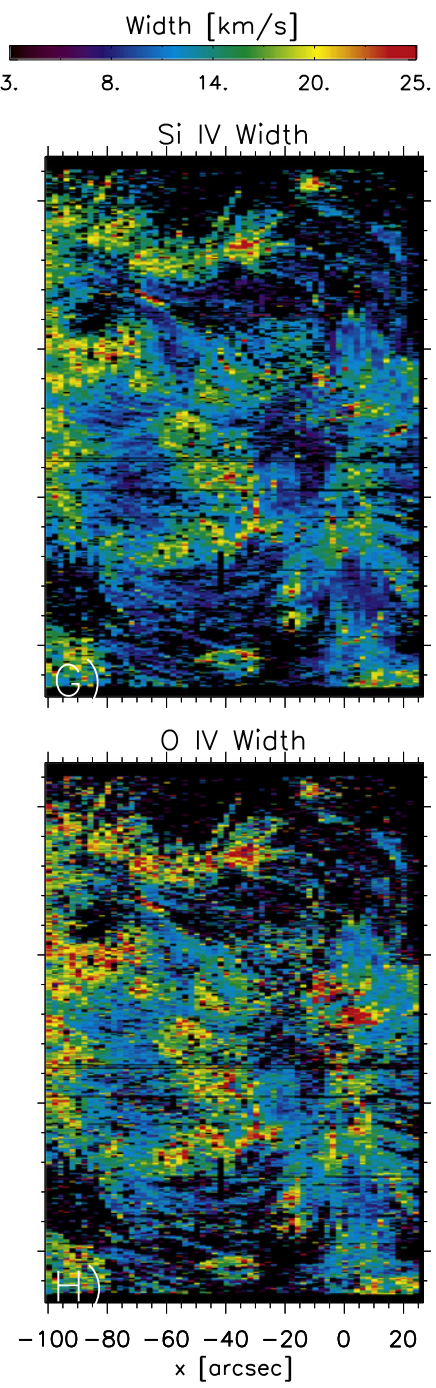

Figure 4. Same layout as Figure 2 for the active region AR1. The SJI 1400 map is at $t=15: 53: 44$ UT (see SJI 1400 Movie 4).

(An animation of this figure is available.)

\subsubsection{IRIS Observations of an Emerging and Flaring AR}

The second active region (AR2) shown in Figure 5 shows microflaring events, highly dynamic jets, and bright loops (see SJI movies available at the IRIS quick look webpage and the online Movie 5 for the SJI 1400). During the raster, the slit in this region crosses loops as they become really bright in $\mathrm{Si}$ IV, such as around $\left[-320^{\prime \prime}, 110^{\prime \prime}\right]$ (Figure 5). The intensity ratio of $\mathrm{Si}$ IV to O IV of these brightening loops is rather low compared to many other regions with even lower intensity in $\mathrm{Si}$ Iv, i.e., the intensity ratio in the brightening loops is low compared to what you would have expected if there was a positive correlation between the $\mathrm{Si}$ IV intensity and the SiIv/ $\mathrm{O}$ IV intensity ratio.

\subsubsection{Intensity Ratio of Si IV to O IV}

One way to visualize the dependence of the intensity ratio of $\mathrm{Si}$ IV to O IV on the properties of the spectral line such as intensity (top row), Doppler shift (middle row), and line widths (bottom row) is with the 2D histograms shown in Figure 6. The top panels reveal one similarity: the intensity ratio between these lines is dependent on their intensity, and in all cases the intensity ratio increases with $\mathrm{Si}$ IV intensity. However, the averages and correlations differ between the different observations and features. Note that the selected targets have different mean intensity ratio values (solid red vertical lines): The coronal hole and quiet Sun data have mean intensity ratio values in the range [3.9-5] (i.e., $\sim[0.6-0.7]$ in logarithmic scale), while the plage and both active region rasters have mean intensity ratios close to 10 ( 1 in logarithmic scale); the quiescent AR1 has a mean intensity ratio of $7(\sim 0.85$ in logarithmic scale), while P11 and AR2 have mean intensity ratios of roughly $\sim 0.95$ in logarithmic scale.

For QS1 and $\mathrm{CH} 1$, the 2D histograms show large similarities, and both reach smaller intensity ratios and intensities than P11, AR1, and AR2. For QS1 and CH1 the intensity ratio correlates with the intensity in a similar manner. Both show an inclined triangle shape, which is due to the fact that the strongest $\mathrm{Si}$ Iv intensities come from features that have only moderate intensity ratios, as mentioned above. The intensity ratio increases more with the Si Iv intensity for the AR than for QS1 and CH1. For the active regions and plage, 


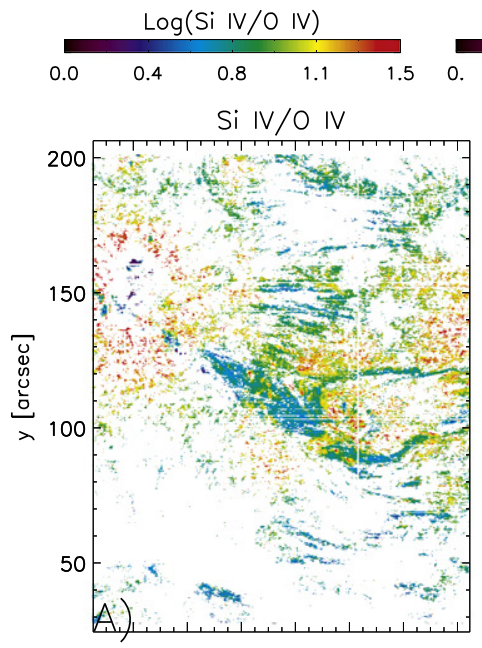

SJI 1400

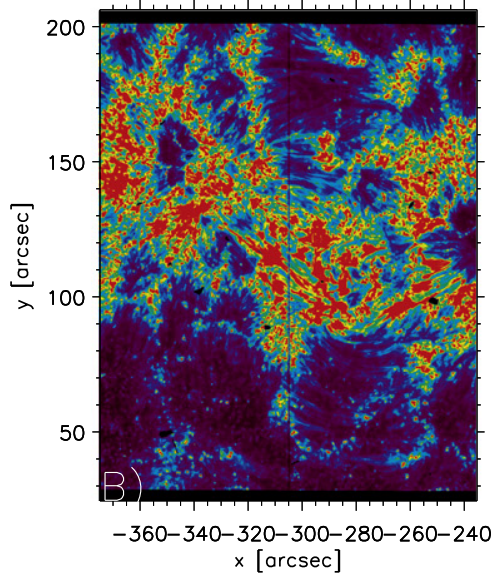

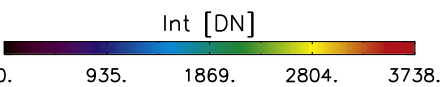

Si IV Int

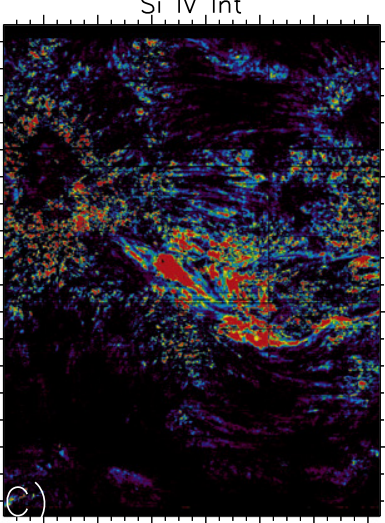

O IV Int

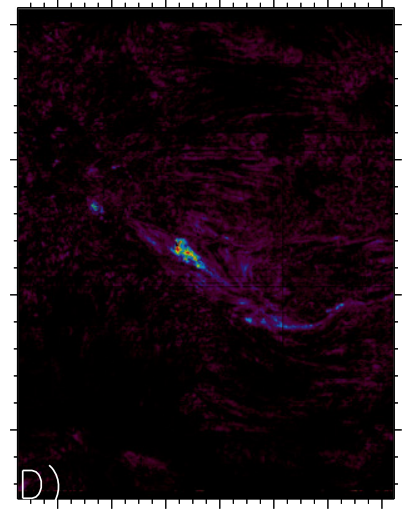

$-360-340-320-300-280-260-240$

$\times[\operatorname{arcsec}]$
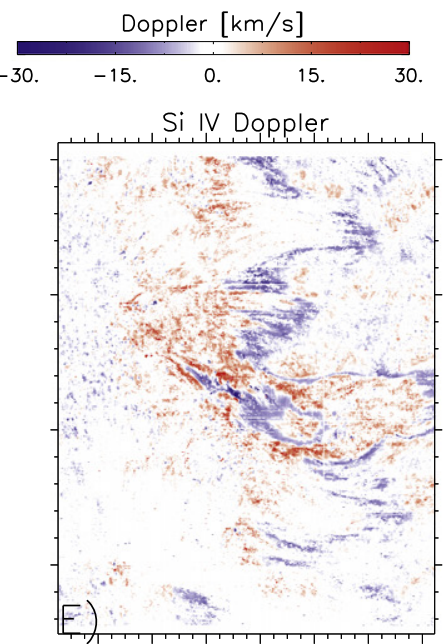

O IV Doppler

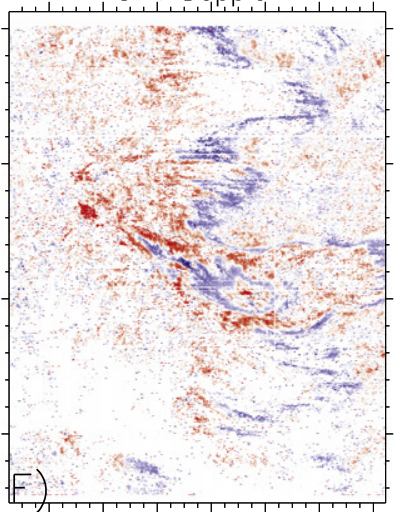

$-360-340-320-300-280-260-240$ $\times[\operatorname{arcsec}]$

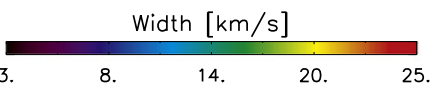

Si IV Width

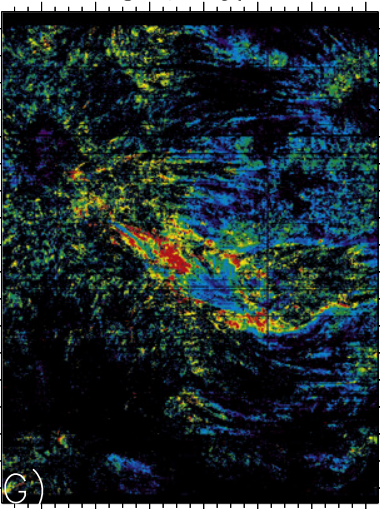

O IV Width

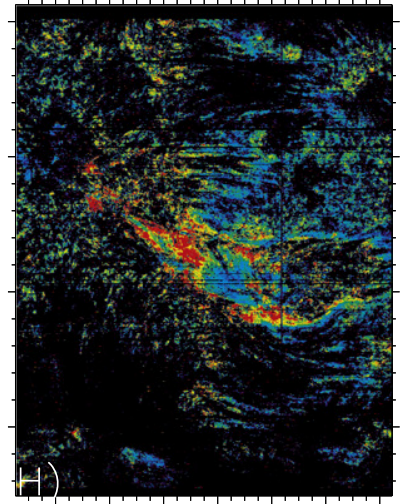

$-360-340-320-300-280-260-240$ $x$ [arcsec]

Figure 5. Same layout as Figure 2 for the active region AR2. The SJI 1400 map is at $t=(+1)$ 00:23:23 UT (see SJI 1400 Movie 5).

(An animation of this figure is available.)

for the same intensity ratio values, the intensity distribution is broader for the flaring and highly dynamic AR (AR2, which shows evidence of emergence, strong flows, jets, flaring, etc.) than for a calm AR (AR1 and Pl). In Pl1, the 2D histogram (panel (C1)) shows some sort of oval structure compared to the triangle shape for QS1 (panel (A1)) and CH1 (panel (B1)). In addition, the umbra observed in P11, AR1, and AR2 corresponds to the tail in the lower end of intensity ratios with rather high $\mathrm{Si}$ IV intensity of the 2D histograms in panels (C1), (D1), and (E1) in Figure 6.

The intensity ratio of Si IV to O IV does not show any clear dependence with the Si Iv Doppler shift in the 2D histograms in Figure 6, or a difference between the various targets. Only the Pl1 region seems to show a faint dependence of the Doppler shift with the intensity ratio. At low intensity ratios there is a small tendency of having positive (red, downward) Doppler shifts and at high intensity ratios a small tendency of negative (blue, upward) Doppler shifts. The intensity ratio of $\mathrm{Si}$ IV to $\mathrm{O}$ IV shows a small increase with increasing Si IV line width in all the observations. In the two ARs and plage, the 2D histograms show a small thin tail at low intensity ratios with rather low line widths and Doppler shifts, which corresponds to the umbra (panels (C2), (C3), (D2), (D3), (E2), and (E3)).

In short, different structures and regions show different intensity ratios; in general, there is a trend of increasing $\mathrm{Si}$ IV/
O IV intensity ratio with $\mathrm{Si}$ IV intensity, but this strongly depends on the observed feature as listed above. In summary, the QS1 and CH1 where they are dominated by acoustic shocks, magnetic elements, or bright points reveal an increase in the intensity ratio with Si IV intensity. However, the increase of the intensity ratio with $\mathrm{Si}$ IV intensity has exceptions such as the brightest points in QS1 and CH1 (Section 4.1.1). The dynamic fibrils and penumbral filaments, seen in the plage and ARs, show a nice correlation between the intensity ratio and $\mathrm{Si}$ IV intensity. Moreover, the dynamic fibrils in the plage seem to have a small correlation between the Doppler shift and the intensity ratio. In active regions, the umbra and flaring loops show relatively low intensity ratios, especially since they are bright in Si IV when comparing to the behavior of bright Si IV features in other regions.

\subsection{Simulations: Synthetic Observations}

What can lead to this correlation between Si IV intensity and $\mathrm{Si}$ IV/O IV intensity ratio? In order to investigate this question, we will use synthetic observations calculated from numerical models. The total intensity of transition region lines such as those of Si IV and O IV is a result of the background plasma state (temperature and density structure, etc.), element abundance, 

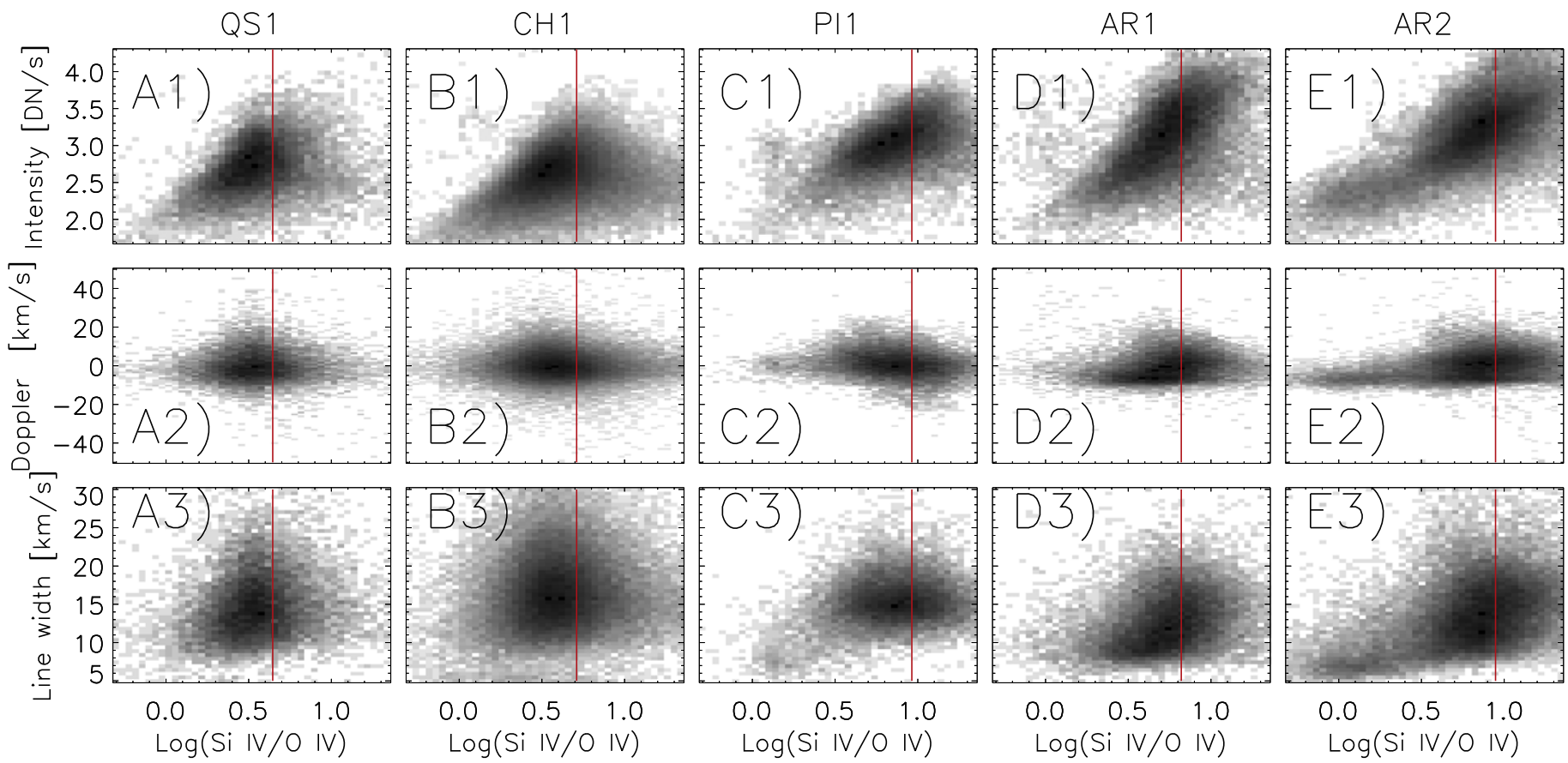

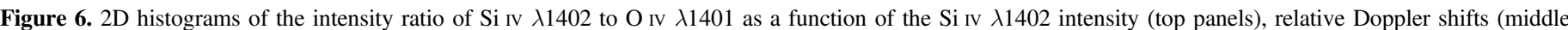

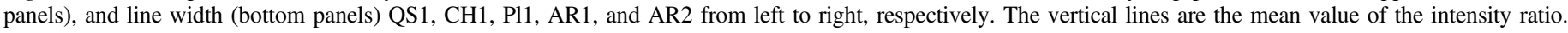

and ionization state of the relevant elements (see the calculations and equations in Section 3.1).

In the following we will treat the background atmospheric model as given and investigate the effects of varying the ionization state (e.g., SE or non-SE) and the abundance (e.g., coronal or photospheric abundances). We describe and discuss the simulated features and the limitations of this model in Section 5, as well as in the result section.

It turns out to be difficult to reproduce both the O IV $\lambda 1401$ and Si IV $\lambda 1402$ intensities when using an SE ionization state and photospheric abundances. This is true for any type of model, including semiempirical models and 1D and 3D radiative MHD simulations (e.g., Olluri et al. 2015). As an example we show intensities calculated based on our 2D model, assuming SE and Grevesse \& Sauval (1998) photospheric abundances (Scenario 1), and compare them with observed quiet-Sun intensities in Figure 7. We find that the $\mathrm{O}$ IV $\lambda 1401$ intensity is larger than Si IV $\lambda 1402$ in the synthetic profiles of scenario 1 , which is the opposite of what we find in the observations. On the other hand, using the same abundances as those derived by Olluri et al. (2015), i.e., using Asplund et al. (2009) photospheric abundances for oxygen and coronal abundances for silicon (Feldman 1992) while retaining the assumption of SE, we find profiles and intensities in closer agreement with the observations, but still the improvement is not good enough (dashed black line, scenario 2). Note that the combination of scenario 2 and non-SE is necessary in order to reproduce the observations (see Section 4.2.2 and Olluri et al. 2015). Therefore, in order to reproduce diagnostics closer to the observations, we will from now on use the set of abundances of scenario 2, unless otherwise mentioned. Even though the average model intensities do not match the observations using SE, it is interesting to see whether we can reproduce the correlation between the ratio of Si IV to $\mathrm{O}$ IV and the $\mathrm{Si}$ IV intensity found in the observations (Section 4.2.1). The

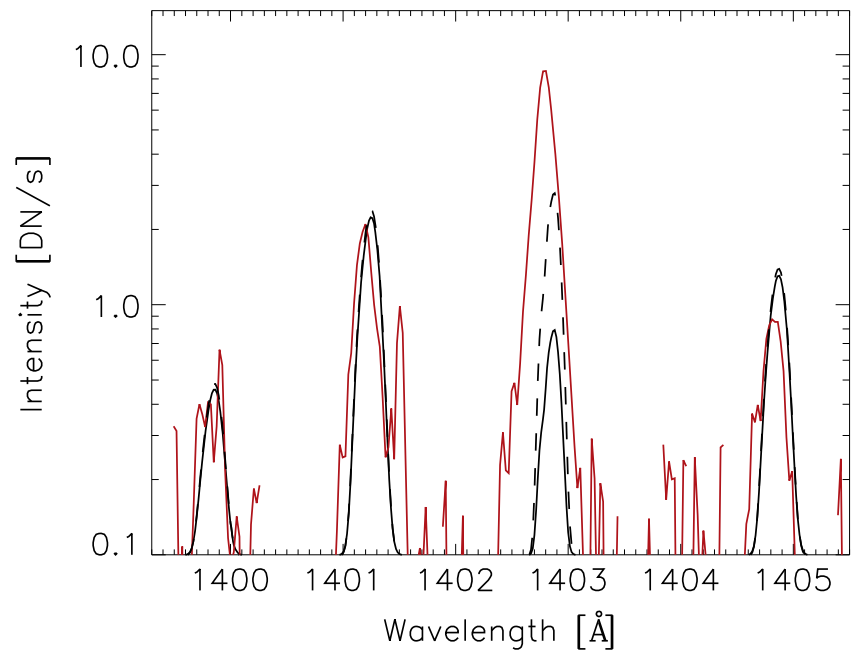

Figure 7. Intensity profiles as a function of wavelength are averaged in time and space for the quiet-Sun target Q1 (solid red line), synthetic profiles using SE approximation and Grevesse \& Sauval (1998) photospheric abundances (solid black line), and following the Olluri et al. (2015) set of abundances (dashed black line).

impact of non-SE effects on the ratio of Si IV and O IV intensity is detailed in Section 4.2.2.

\subsubsection{Statistical Equilibrium}

The 2D histogram of the ratio of the $\mathrm{Si}$ IV $\lambda 1402$ to O IV $\lambda 1401$ intensities as a function of $\mathrm{Si}$ IV $\lambda 1402$ intensity for scenario 2 (top panel of Figure 8) shows a variation of the ratio dependent on the intensity. However, the mean value of the intensity ratio is almost five times smaller than in the observations. Furthermore, the correlation of the Si IV intensity with the intensity ratio is not the same for the SE simulations and the observations (top panels of Figure 6). In this plot we have degraded the synthetic data in order to take into account 

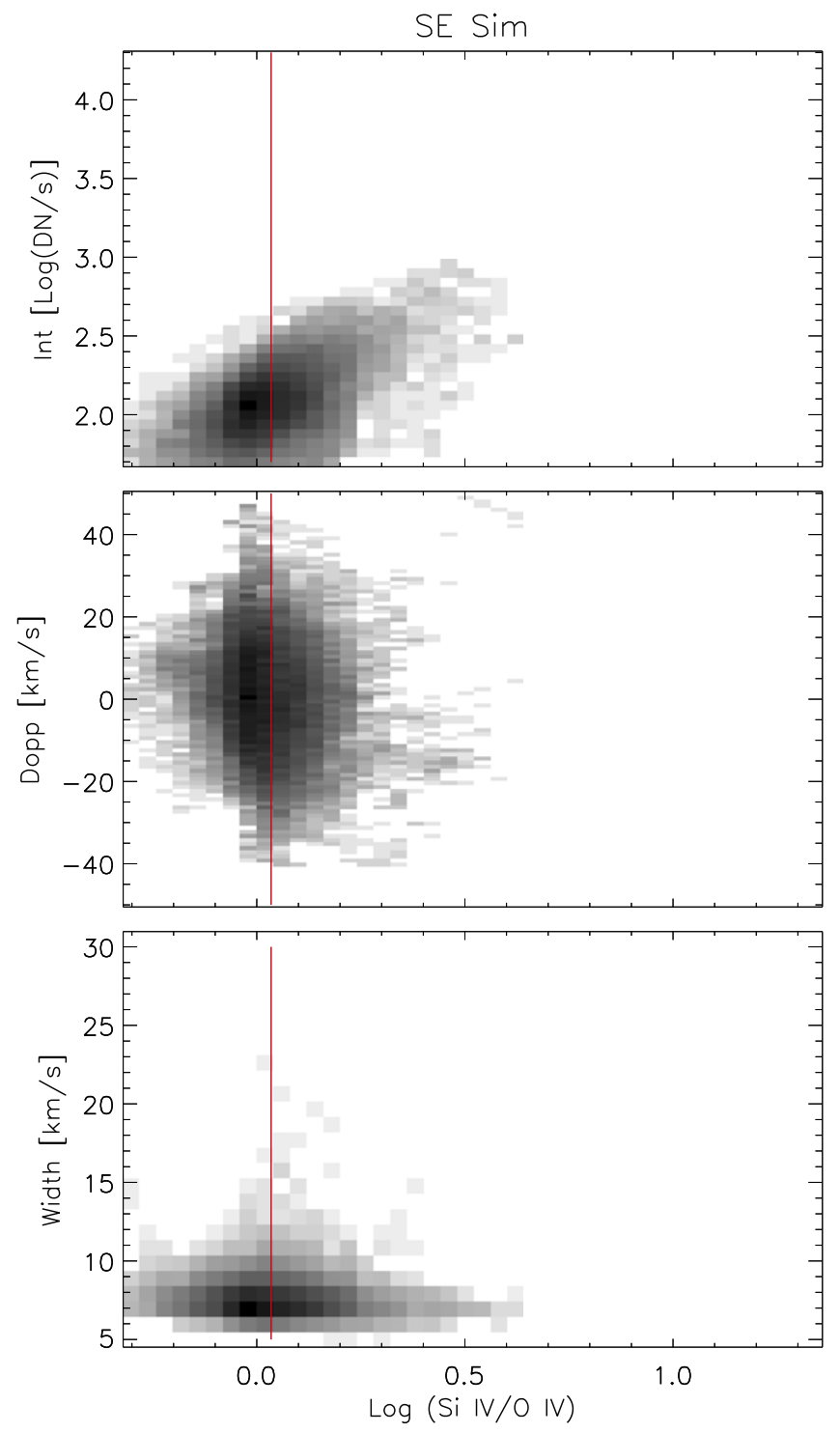

Figure 8. 2D histograms of the ratio of Si IV $\lambda 1402$ to $\mathrm{O}$ IV $\lambda 1401$ intensities as a function of the Si IV $\lambda 1402$ intensity (top), Doppler shifts (middle), and line width (bottom) for synthetic SE case. The vertical lines are the mean value of the intensity ratio.

the finite IRIS spatial and temporal resolution to allow a better comparison with the observations.

The Doppler shift histogram (middle panel of Figure 8) shows a larger range of velocities than any of the observations (compare with Figure 6), most likely owing to the simplified magnetic field configuration of the simulation. The magnetic field in the model is mostly unipolar and vertical. This leads to larger Doppler shifts since most of the flows are along the magnetic field lines that are aligned with the LOS integration. This is in contrast to the observations, where the LOS is not necessarily aligned with the flows and/or the magnetic field. The ratio of $\mathrm{Si}$ IV to O IV intensities does not show any clear dependence on the Si IV Doppler shift in the 2D histogram, which is similar to what we find in the observations (Figure 6).

The line width is smaller in the simulation than in the observations (compare bottom panels of Figures 6 and 8), most likely because our model does not have enough small-scale dynamics owing to the lack of type II spicules, small- and large-scale flux emergence, partial ionization effects, etc. We do not find any correlation between the line width and the intensity ratio of $\mathrm{Si}$ IV to $\mathrm{O}$ IV, in contrast to the observations, which show an increase of the ratio when the line width increases.

As mentioned above, the intensity ratio does not follow the same dependence as found in the observations, but it does at least show some variation with the intensity. Let us consider the cause of this variation. First of all, the logarithm of the peak formation temperature (in SE) of $\mathrm{O}$ IV is 5.2 and that of $\mathrm{Si}$ IV is 4.8. Therefore, the emission in each voxel of the simulation will be different for $\mathrm{O}$ IV and Si IV, depending on the temperature. In addition, the source functions $G\left(T, n_{e}\right)$ for both lines are sensitive to density around $8 \times 10^{4} \mathrm{~K}$ (see, e.g., Grevesse \& Sauval 1998). One way to visualize the dependence of the emission on temperature is shown in Figure 9. This figure shows 2D histograms of the emission of O IV (left panel) and Si IV (middle panel), both as a function of temperature. The $2 \mathrm{D}$ histogram of the ratio of $\mathrm{Si} I V$ to $\mathrm{O}$ IV emission as a function of temperature is shown in the right panel. Note that the intensity ratio is nicely correlated with temperature. However, the correlation becomes weaker at temperatures between $\log (T)=[4.75,5.05]$. In this range, the source functions $G(T)$ of both lines are density sensitive. As a result, in the same temperature range, the $2 \mathrm{D}$ histogram takes on the apparent shape of a cross rotated counterclockwise some $30^{\circ}$. This means that the ratio becomes double valued and the 2D histogram has two peaks at two different intensity ratios, e.g., at $\log (T)=4.8$ the histogram peaks both at an Si IV/ $\mathrm{O}$ IV intensity ratio of 1.95 and at 2.3 .

As a result of the variation of the ratio of $\mathrm{Si}$ IV to $\mathrm{O}$ IV intensity with temperature, any variation of the density stratification within the transition region may produce a different intensity ratio. This is illustrated using a toy model and shown in Figure 10. In this toy model (and the figure) we synthesized Si IV and O Iv profiles using SE and Grevesse \& Sauval (1998) photospheric abundances. We used photospheric abundances because the intensity ratio of $\mathrm{Si}$ IV to $\mathrm{O}$ IV differs the most from the observations in this case. As a result, this case of photospheric abundances is ideally suited to illustrate and enhance the impact of density stratification changes on the intensity ratio. The temperature stratification that we used to synthesize these profiles is based on taking averages in time and across horizontal cuts of our model (dotted line in the right panel). Each line profile is for an accompanying density stratification (marked with the same color in the solid lines of the right panel). The emergent synthetic profiles are shown in the left panel following the same color scheme as in the right panels, and all of them have been normalized to the peak intensity of O IV $\lambda 1401$. We overplot for comparison with dashed lines the observed mean profile for the QS1 (black) and AR1 (red) targets. Note that one finds intensity ratios similar to those observed with a specific density and temperature stratification, without changing the values of the abundances or using non-Gaussian profiles (Dudík et al. 2014). The stratifications chosen are unrealistic, and we do not believe that the solution to our problem lies in choosing the stratification that comes "closest" to the observed case. Rather, the purpose of this figure is to visualize the impact of different stratifications on the line intensity ratios. The black-blue curves indicate models in which the density is high and where it decreases slowly with height, also in the temperature range that covers the contribution functions of $\mathrm{O}$ IV and SiIV. The green-yellow-red curves 

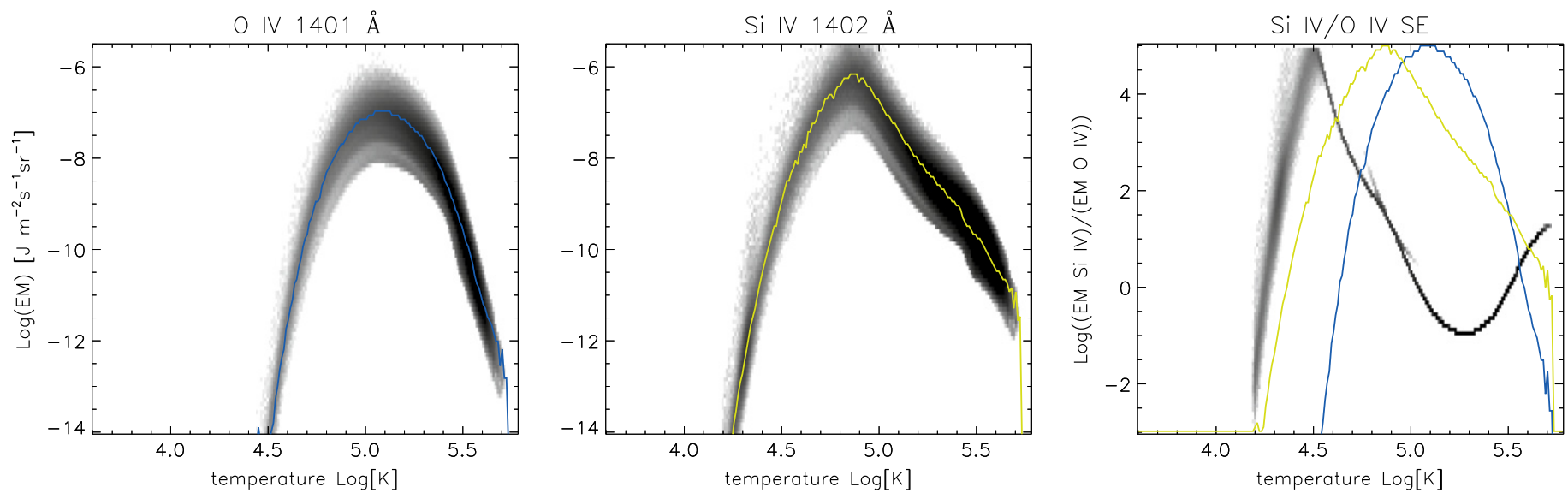

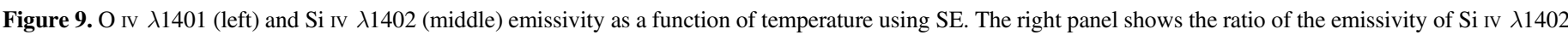

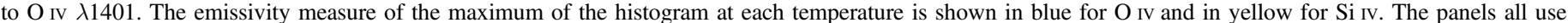
logarithmic axes.
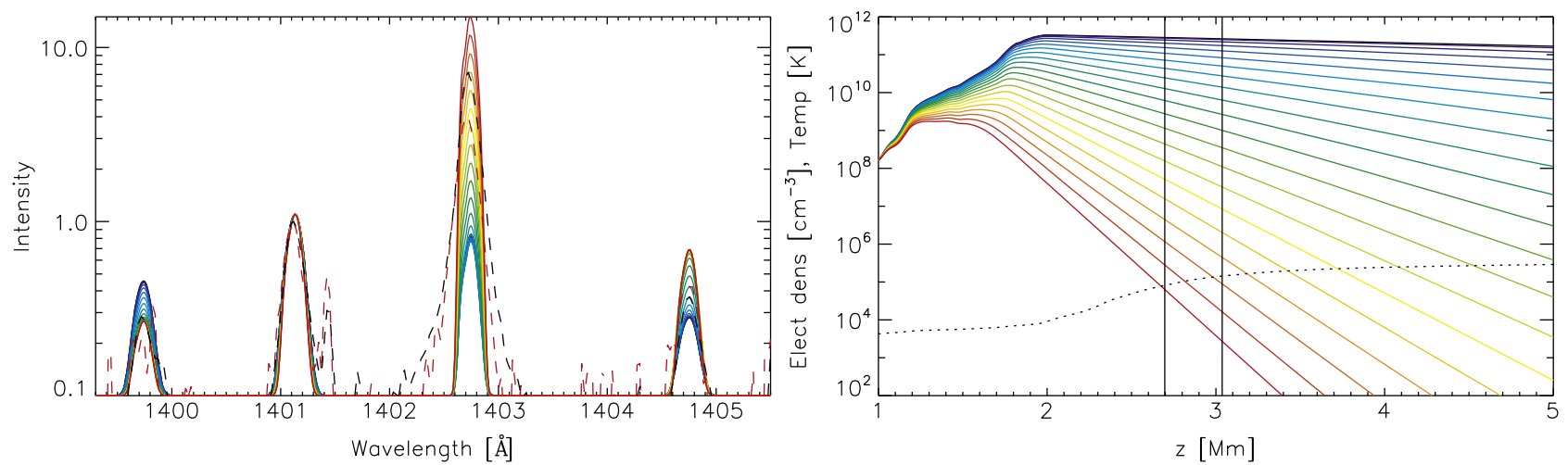

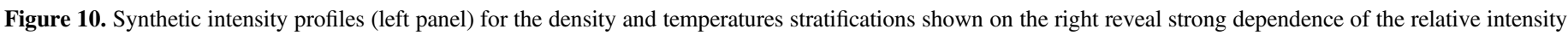

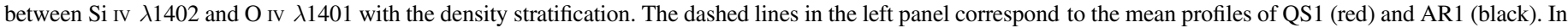

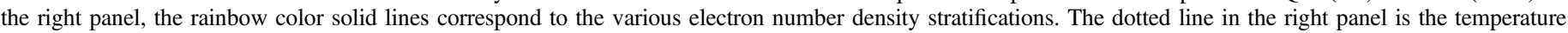

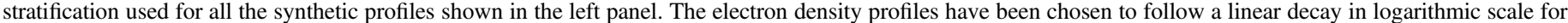
the total mass density. The vertical lines in the right panel are the formation temperature of Si IV (left) and O IV (right).

indicate models where the density is lower and decreases more rapidly with height across the relevant temperature range. In these simplified scenarios (green-yellow-red curves) the increase in the intensity ratio of $\mathrm{Si}$ IV to $\mathrm{O}$ IV with $\mathrm{Si}$ IV intensity is a result of a fast density decrease with height in the relevant temperature range. However, for the highest densities (blueblack curves) the behavior of the intensity ratio of $\mathrm{Si}$ IV to $\mathrm{O}$ IV is the opposite, and the ratio increases with increasing density, i.e., the slope is not highly relevant for these cases. This is due to the fact that the source functions for these lines are density sensitive for the high-density cases (black-blue curves).

The correlation between the $\mathrm{Si}$ IV/O IV intensity ratio and $\mathrm{Si}$ IV intensity is related to varying density stratifications in the transition region (Figure 11). The time evolution of this figure is shown in the corresponding Movie 1. The figure and movie show the temperature (top left panel), vertical velocity (bottom left panel), and emission of Si IV $\lambda 1402$ using SE (top middle panel) and non-SE (bottom middle panel) and of O IV $\lambda 1401$ using SE (top right panel) and non-SE (bottom right panel) at one specific instant. We overplot the intensity of Si IV $\lambda 1402$ for SE (top middle panel) and non-SE (bottom middle panel) and of O IV $\lambda 1401$ for SE (top right panel) and non-SE (bottom right panel) with white dashed lines and the intensity ratio between them (solid lines) assuming SE (top panels) and non-SE (bottom panels). The temperature contour at $10^{5} \mathrm{~K}$ is shown in the bottom left panel in green. The computed emission and intensity are convolved in space and time with the spatial and temporal resolution of the IRIS observations used in this work. Leaving the non-SE case for Section 4.2.2, we find that for SE the stronger the intensity in $\mathrm{Si}$ IV, the higher the intensity ratio. This is because for bright locations, the emission in Si IV spreads over a larger range of heights along the LOS, similar to the behavior observed in the toy model described in Figure 10. In contrast, O IV emission also spreads over a greater range of heights in these regions, but is less enhanced. The largest values of the intensity ratio are located at the side boundaries of the dynamic fibrils (i.e., incursions of elongated structures of the TR into the corona; Hansteen et al. 2006) that are aligned with the LOS, i.e., along the vertical axis. In such locations $\mathrm{Si}$ IV emission is larger than elsewhere. It is this type of stratification that seems to be the most favorable in terms of having larger intensities in $\mathrm{Si}$ IV than in O IV. This is because the boundary of the extended structure along the LOS has temperatures closer to the formation temperature of $\mathrm{Si}$ IV than $\mathrm{O}$ IV.

Another physical process that leads to an increase of the intensity ratio of $\mathrm{Si}$ IV to $\mathrm{O}$ IV is when magnetoacoustic shocks pass through the transition region. This enhances the density around the formation temperature of Si IV more than around the formation temperature of $\mathrm{O}$ IV owing to the density drop in the 


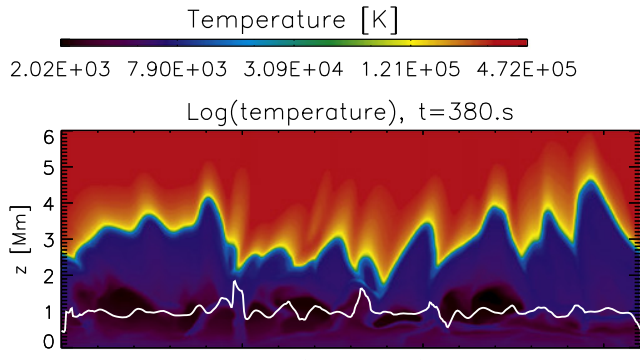

Vertical velocity

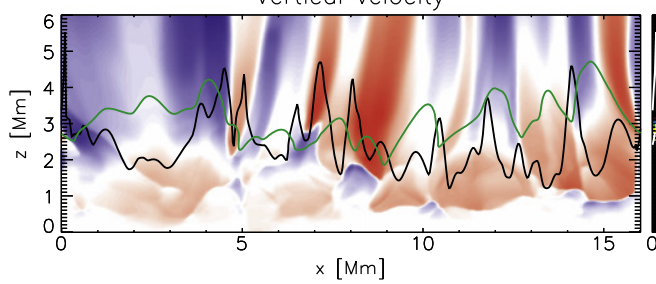

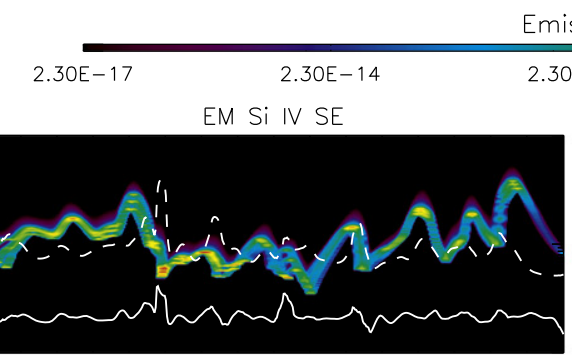

EM Si IV non-SE

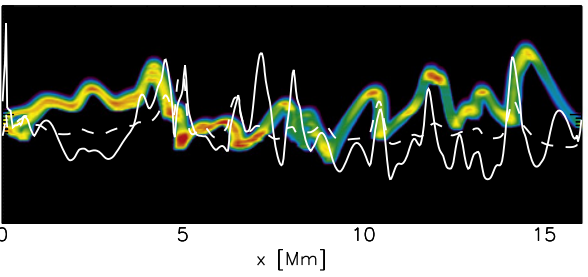

mission

$\begin{array}{lll}2.30 E-08 & 2.30 E-05\end{array}$

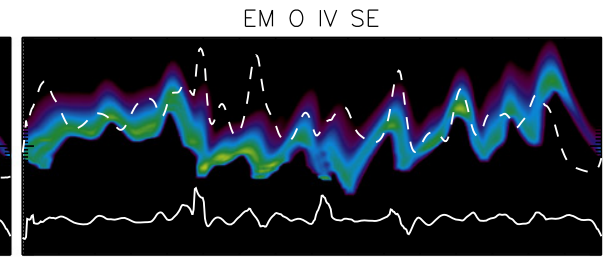

EM O IV non-SE

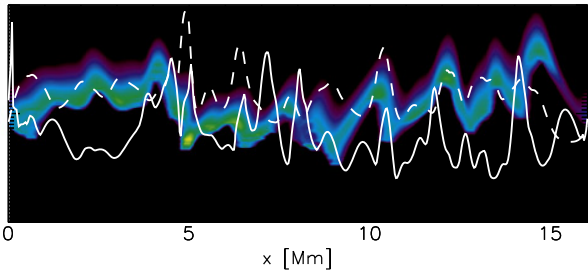

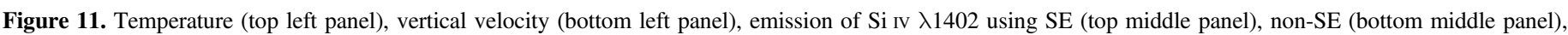

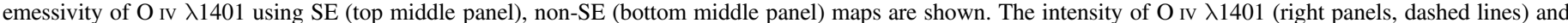

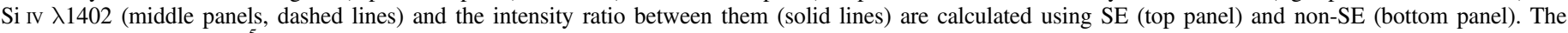

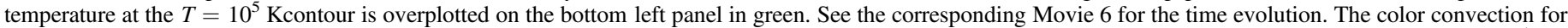
the vertical velocity follows the same as the Doppler shift convection, i.e., upflows are in blue and downflows are in red, and ranges within [-40, 40$]$ km ${ }^{-1}$.

(An animation of this figure is available.)

transition region (e.g., as near $x=8.3 \mathrm{Mm}$ in Figure 11). In the online supporting Movie 6 one can appreciate that this process lasts only a short period of time $(\leqslant 10 \mathrm{~s})$, i.e., the time it takes the shock to go through the transition region.

\subsubsection{Nonstatistical Equilibrium}

The synthesis of silicon and oxygen emission from 2D radiative MHD simulations when taking into account timedependent nonequilibrium ionization reduces the discrepancy between the observed and simulated intensity ratios of $\mathrm{O}$ IV to Si IV. Olluri et al. (2015) managed to match the synthetic and observed intensity ratios assuming Asplund et al. (2009) photospheric abundances for oxygen and coronal abundances for silicon (Feldman 1992), as well as non-SE ionization in 3D simulations. We are able to reproduce these results, as shown in Figure 12, which compares synthetic and observed QS1 line profiles (compare the SE results shown in Figure 7). For comparison we overplot the profiles using photospheric abundances (Grevesse \& Sauval 1998) but retaining non-SE ionization (solid black line). Only using non-SE and abundances from Grevesse \& Sauval (1998) is not enough to match the observations, though it is better than the SE case (Figure 7). In this $2 \mathrm{D}$ radiative MHD simulation, the non-SE ionization states of silicon and oxygen are important owing to the highly dynamic state of the transition region plasma that is being heated and cooled continuously, as presumably is the case for the real Sun.

Thus, the total average synthetic profiles give a good match with observations using the abundances recommended by Olluri et al. (2015) and non-SE ionization. However, the question remains whether the variation of the intensity ratio of $\mathrm{Si}$ IV to $\mathrm{O}$ IV as a function of their intensities is reproduced. The 2D histogram of the ratio of the emission of Si IV $\lambda 1402$ to O IV $\lambda 1401$ for the synthetic non-SE case as a function of Si IV $\lambda 1402$ intensity (top panel of Figure 13) shows that the intensity ratio is dependent on the intensity and that the resemblance with observations has improved significantly

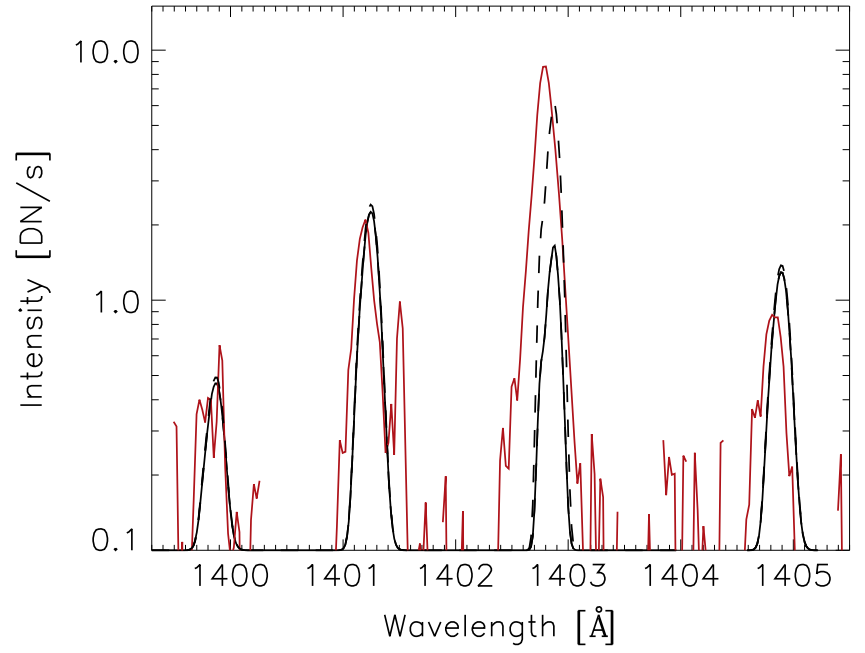

Figure 12. Intensity profiles as a function of wavelength are averaged in time and space for QS1 (solid red line), synthetic profiles using non-SE approximation and Grevesse \& Sauval (1998) photospheric abundances (solid black line), and the following Olluri et al. (2015) set of abundances (dashed black line).

compared to the SE case (see Figures 6 and 8). The 2D histogram between the intensity ratio and the Si IV intensity shows an inclined oval shape, rather similar to the plage observations, though it does not reproduce the triangle shape of the observed QS1 and CH1 (Figure 6).

Another interesting aspect is that the Doppler shift, in contrast to most of the observations and SE, shows a variation as a function of the ratio of Si IV to $\mathrm{O}$ IV intensity. At low intensity ratios, the Doppler shift tends to be positive, and at high intensity ratios, it tends to be negative. This, again, has some similarity with the plage Pl1 observations despite the obvious differences between the weak magnetic field in the simulation as compared to the presumably much stronger field in plage regions. The resemblance may be due to the fact that in 

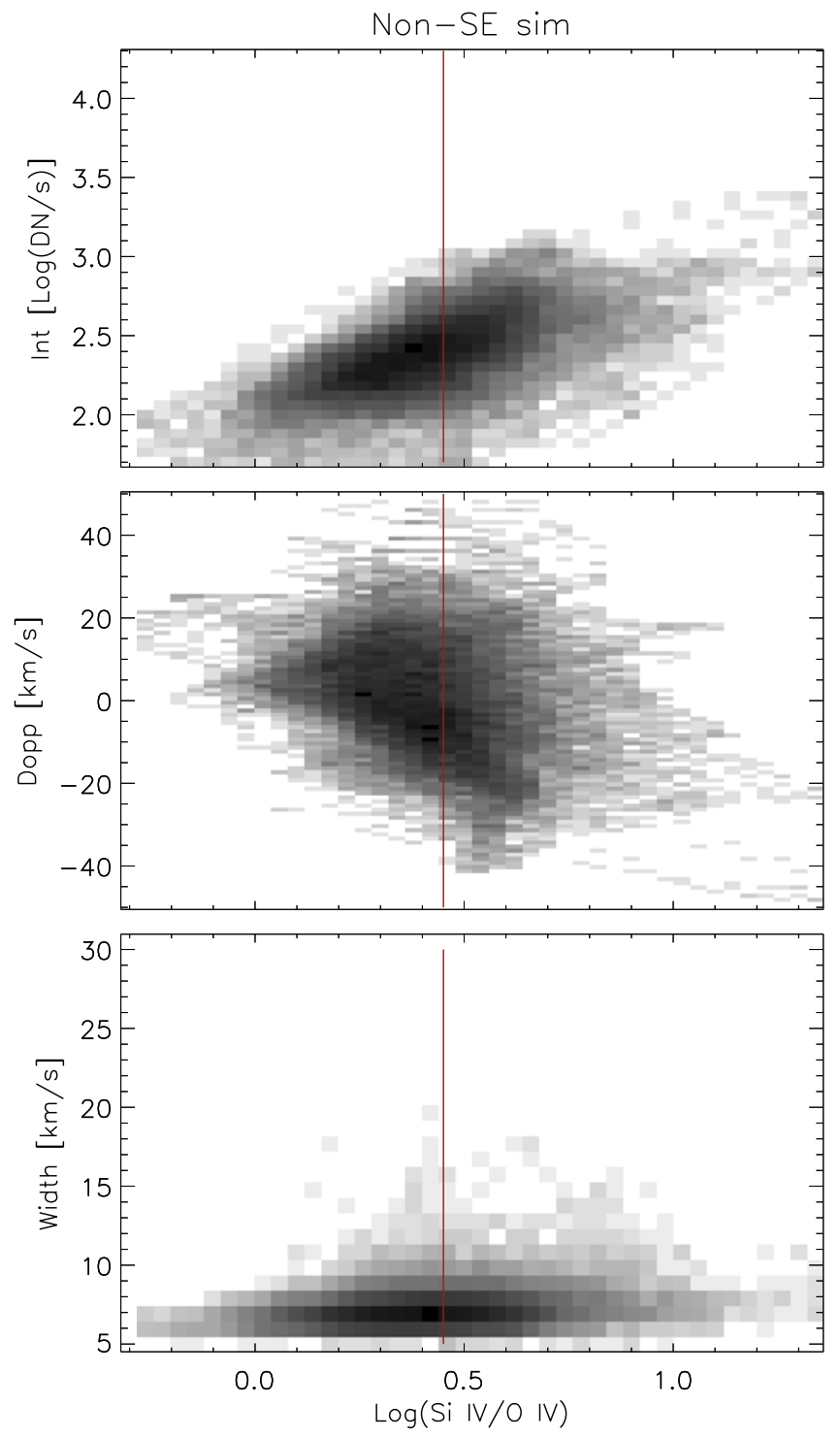

Figure 13. Same layout as in Figure 8 using non-SE.

P11 the magnetic field has a preferential direction that may be roughly aligned with the LOS. This is because 2D simulations in some sense are more plage-like since the expansion of flux tubes is restricted to two dimensions and the initial setup does not include closed magnetic field loops. In the plage there is also less expansion with height of flux tubes because of the stronger fields. The intensity ratio has a small dependence on the line width (similar to the observations), whereas for SE we do not find any correlation. In addition, the line width for the non-SE case is somewhat broader than the SE cases, but is still small compared to the observations.

Non-SE adds an extra complexity into the comparison between Si IV $\lambda 1402$ and O IV $\lambda 1401$. In non-SE, the emission of Si IV and O IV is spread over a wider range of temperatures than when assuming SE (compare left and middle panels of Figures 9 and 14). In addition, the intensity ratio between these two lines is a nonunique function of temperature, in contrast to the SE case (compare right panels of Figures 9 and 14). In non$\mathrm{SE}$, within the same temperature bin, the intensity ratio spreads over a large range of values. Still, a strong dependence on temperature is found: at low temperatures the intensity ratio decays several orders of magnitude. For higher temperatures, above $\log (T)=4.7$, the intensity ratio increases with temperature.

Which physical processes lead to the correlations of Figure 13? Figure 15 shows the intensity of Si IV (panel (A)), the intensity ratio of Si IV and O IV in non-SE (panel (B)) and $\mathrm{SE}$ (panel (C)), and the ratio of the total mass of the $\mathrm{Si}^{3+}$ and $\mathrm{O}^{3+}$ (panel (D)). In general, we find that the stronger the Si IV intensity (panel (A)), the stronger the intensity ratio (panel (B) and $(\mathrm{C})$ ), although there is no one-to-one correlation. In addition, many features observed in the maps of the intensity ratios in SE (Panel (C)) and non-SE (panel (B)) do not resemble each other. Since the intensity ratio in SE is directly related to the density stratification in the transition region (Figure 10), the intensity ratio for non-SE does not seem to be as well correlated (as in the SE case) with the density stratification between the temperatures of maximum formation of $\mathrm{Si}$ IV and $\mathrm{O}$ IV. Of course, this results from the fact that the ratio of the total mass of the $\mathrm{Si}^{3+}$ and $\mathrm{O}^{3+}$ using non-SE (panel (D)) differs from the ratio assuming $\mathrm{SE}$.

Let us describe what leads to the various intensity ratios in the simulation for the non-SE case, since it is obvious that it follows certain features in space and time. First, let us focus on the typical evolution of a spicule; we have a clear example around $x=3 \mathrm{Mm}$ and $t=[650,680] \mathrm{s}$ shown in Figure 15. In the non-SE case, the intensity ratio between $\mathrm{Si} I V$ and O IV decreases in time gradually, whereas in the SE case, there is a rather high intensity ratio just at the beginning of the evolution, and after it, the intensity ratio remains almost constant and low. The gradual decay with time of the intensity ratio for the non-SE case leads to the correlation of the intensity ratio with the Doppler shift seen in Figure 13. This is in contrast to the SE case, for which the intensity ratio does not show any dependence on the Doppler shift. At the earlier stages, the spicules move upward, showing negative Doppler shifts and high intensity ratios, and at the later stages they move downward, with positive Doppler shifts and small intensity ratios. The gradual decay in the intensity ratio for the non-SE case is a direct consequence of a slow decay in the total mass ratio of $\mathrm{Si}^{3+}$ and $\mathrm{O}^{3+}$, i.e., $\mathrm{Si}^{3+}$ ionizes faster than $\mathrm{O}^{3+}$. The line width tends to increase with the ratio of $\mathrm{Si}$ IV to $\mathrm{O}$ IV intensity owing to the transition region being expanded. Therefore, one may expect a greater range of nonthermal velocities than in other locations.

Another feature that leads to an increase of the intensity ratio in the non-SE case is similar to the case that we described for the SE case, where the intensity ratio depends on the orientation of the LOS integration relative to the spicule axis and the location within the spicules. For the non-SE case we also find that the largest intensity ratios come from colliding spicules (see Movie 6, or around $x=7 \mathrm{Mm}$ in Figure 11) or from two neighboring spicules, one next to the other, with strong opposite flows along the LOS, e.g., around $x=4.5$ in Figure 11. In summary, we find that for the non-SE case the dependence of the intensity ratio on the intensity of Si IV is a result of the thermal and dynamic properties of the atmosphere. This is also true for the SE case, but the main difference is that the dynamic properties of the atmosphere are very different in the non-SE case because of the long ionization and recombination timescales of these ions. 

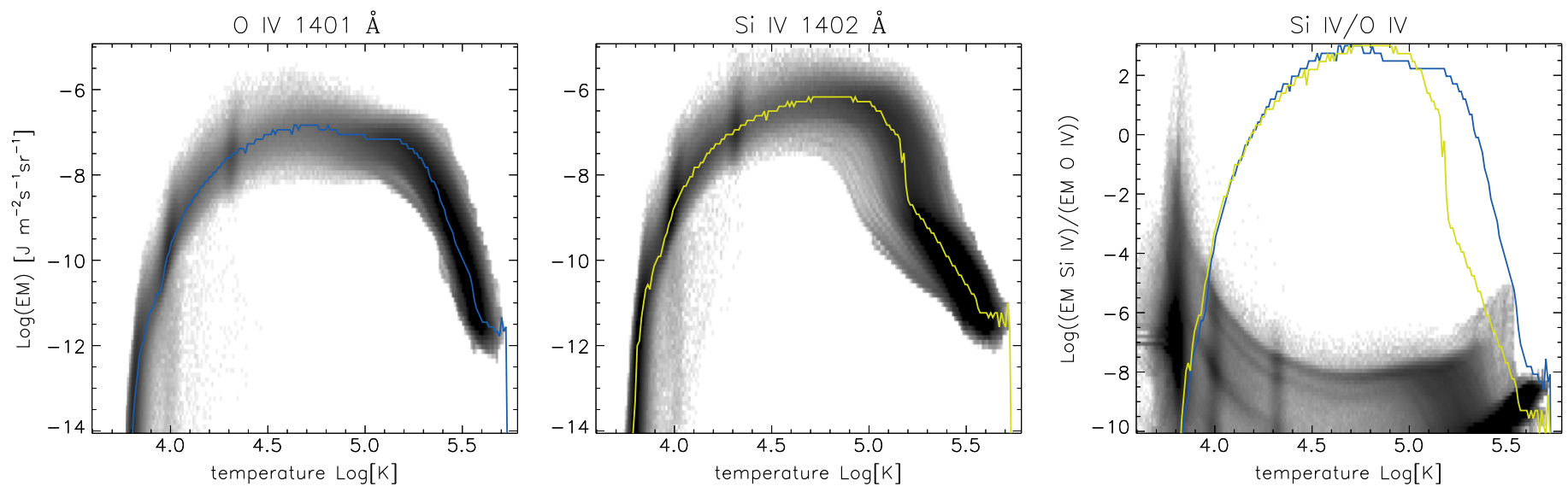

Figure 14. Same layout as in Figure 9 using non-SE.

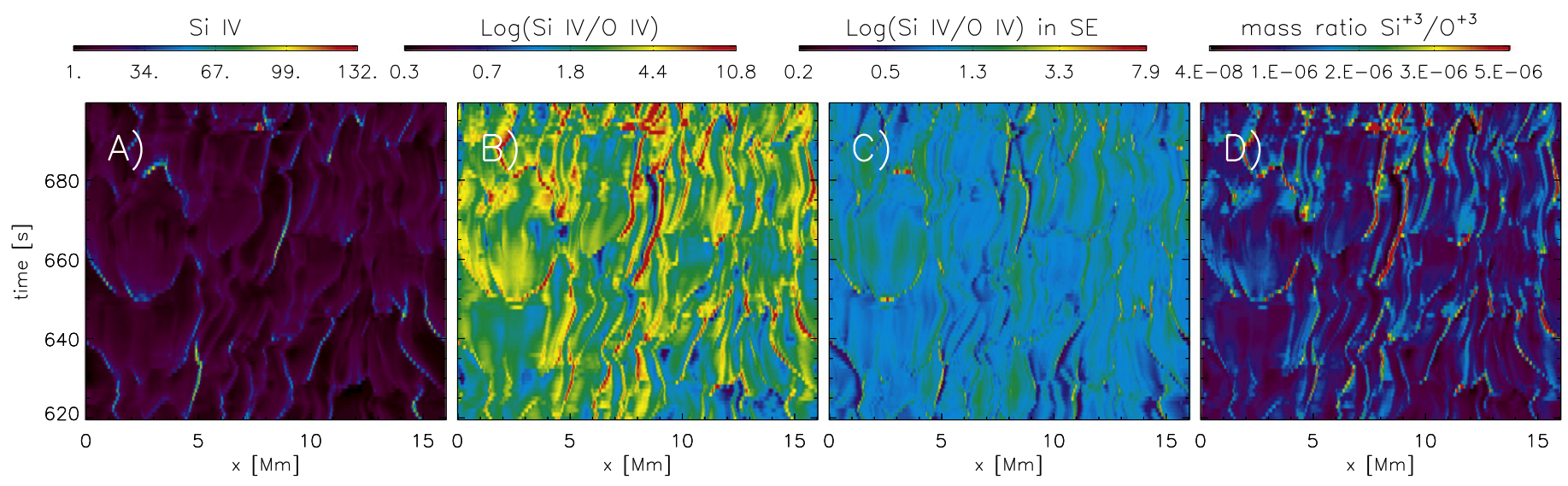

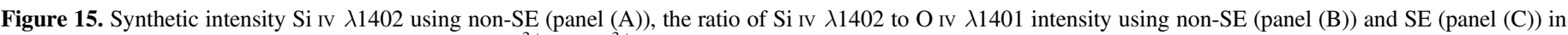
logarithmic scale, and the ratio of the total mass of $\mathrm{Si}^{3+}$ and $\mathrm{O}^{3+}$ using non-SE (panel D) are illustrated as a function of space and time.

\section{DISCUSSION AND CONCLUSIONS}

In this work we combined self-consistent 2D radiative MHD simulations of the solar atmosphere with IRIS observations in order to study the nonequilibrium properties of silicon and oxygen. IRIS is well suited for this study owing to the high cadence, spatial resolution, and high signal-to-noise ratios for the O Iv $\lambda 1401$ and Si Iv $\lambda 1402$ lines. Olluri et al. (2015) were able to match the intensity ratios of averaged spectral profiles in space and time of Si IV $\lambda 1402$ and O IV $\lambda 1401$, taking into account the nonequilibrium effects. Our work analyzes the properties of the intensity ratio of these two lines as a function of space and time. For this, we analyzed different regions on the Sun (QS, CH, plage, and AR) and observed a strong correlation between the ratio of Si Iv to $\mathrm{O}$ Iv and the Si Iv intensity. The intensity ratio values and the correlation vary depending on the observed region and features. We find only a small correlation of the intensity ratio with Doppler shifts. For the line width, the intensity ratio increases with the line width.

The fact that the synthetic observables of Si IV and O IV differ considerably when assuming SE versus non-SE and that the latter reproduces many properties of the observables is a strong indication of the nonequilibrium ionization nature of $\mathrm{O}^{+3}$ and $\mathrm{Si}^{+3}$ for the various observed regions' features on the Sun. We find that the observed dependence with Si IV intensity of the Si IV/O IV intensity ratio can be explained by the interplay between the thermal properties of the stratification, the dynamics of the atmosphere, and the nonequilibrium ionization of $\mathrm{O}^{+3}$ and $\mathrm{Si}^{+3}$. Our results indicate that interpretations based on comparisons between these $\mathrm{O}$ IV and $\mathrm{Si}$ IV lines are risky unless the effects of nonequilibrium ionization are fully considered. This is because these lines are clearly in non-SE, the temperature formation is not exactly the same, and the ratio of Si IV and $\mathrm{O}$ IV intensity spreads over a wide range of values depending on the observed region $(\mathrm{QS}, \mathrm{CH}, \mathrm{Pl}, \mathrm{AR})$ and features (spicule, dynamic loops, umbra, jets, microflares, etc.). Such multiline analysis will be impacted by these processes, which casts doubt on using these lines for constraining abundances (Olluri et al. 2015), density diagnostics, or kappa-functions (Dudík et al. 2014) without including nonSE effects in the interpretation.

The simulations are able to reproduce some of the observables when non-SE and the Olluri et al. (2015) set of abundances are taken into account, i.e., oxygen abundances from Asplund et al. (2009) and "coronal" abundances for silicon (Feldman 1992). We would like to refer to Asplund et al. (2009), Pereira et al. (2013), and Fabbian \& MorenoInsertis (2015) for a deeper discussion on the oxygen and other atmospheric abundances. We note that the rationale for the set of abundances selected by Olluri et al. (2015) (and used here as well) is based on the first ionization potential (FIP) effect, i.e., the fact that low-FIP elements such as silicon tend to be overabundant in the transition and corona.

Our simulations are highly dynamic, are self-consistent, and include many of the physical processes in the chromosphere, 
transition region, and corona, such as radiative transfer with scattering and thermal conduction along the magnetic field lines. However, our simulations are still quite simplified since they are 2D and the magnetic field is vertical and unipolar without magnetic flux emergence (Martínez-Sykora et al. 2008, 2009a). The simulation is also missing physical processes that may play an important role in the chromosphere and transition region, such as partial ionization effects (Martínez-Sykora et al. 2015a) and time-dependent hydrogen (Leenaarts et al. 2007) and helium ionization (Golding et al. 2014), as well as particle acceleration, which may be important in the transition region and corona in physical processes such as magnetic reconnection (Baumann \& Nordlund 2012; Testa et al. 2014). Therefore, our simulations indicate that the intensity ratio as a function of the $\mathrm{Si}$ IV intensity is a consequence of the emission from dynamic fibrils in combination with non-SE effects in these lines. The simulation is dominated by magnetoacoustic shocks going through the transition region (type I spicules; Hansteen et al. 2006), but does not include type II spicules, emerging flux, flaring regions, etc. Therefore, we need to expand this investigation with simulations that can reproduce the missing physics, features, and regions (e.g., $\mathrm{AR}, \mathrm{Pl}$, enhanced network) in order to investigate whether similar correlations between the ratio of Si IV and OIV and the Si IV intensity can be obtained, and whether thermodynamics and non-SE effects play a similar role as in the current simulation. However, we can tentatively extrapolate some of the results of our simulations to the observations. The enhanced plage regions usually are dominated by dynamic fibrils, which is also the case in our simulations. Note that the distribution in the 2D histogram for the non-SE case is oval. This is similar to the Pl1 observations but with lower intensities and intensity ratios for the simulations. Therefore, the distribution of the intensity ratio as a function of the Si IV might be a consequence of the dynamics of the dynamic fibrils in combination with non-SE effects on the lines.

We gratefully acknowledge support by NASA grants NNX11AN98G and NNM12AB40P and NASA contracts NNM07AA01C (Hinode) and NNG09FA40C (IRIS). This research was supported by the Research Council of Norway and by the European Research Council under the European Union's Seventh Framework Programme (FP7/2007-2013)/ ERC Grant agreement nr. 291058. The simulations have been run on clusters from the Notur project and the Pleiades cluster through the computing project s1061 from the High End Computing (HEC) division of NASA. We thankfully acknowledge the computer and supercomputer resources of the Research Council of Norway through grant 170935/V30 and through grants of computing time from the Programme for Supercomputing. We would like to thank the referee for her/ his constructive comments, which have helped us improve the paper. This work has benefited from discussions at the International Space Science Institute (ISSI) meetings on "Heating of the Magnetized Chromosphere," where many aspects of this paper were discussed with other colleagues.

\section{REFERENCES}

Archontis, V., \& Hansteen, V. 2014, ApJL, 788, L2

Asplund, M., Grevesse, N., Sauval, A. J., \& Scott, P. 2009, ARA\&A, 47, 481

Baumann, G., \& Nordlund, Å. 2012, ApJL, 759, L9

Bradshaw, S. J., \& Cargill, P. J. 2006, A\&A, 458, 987

Bradshaw, S. J., \& Klimchuk, J. A. 2011, ApJS, 194, 26

Carlsson, M., Judge, P. G., \& Wilhelm, K. 1997, ApJL, 486, L63

Carlsson, M., \& Leenaarts, J. 2012, A\&A, 539, A39

Carlsson, M., \& Stein, R. F. 1992, ApJL, 397, L59

Carlsson, M., \& Stein, R. F. 1997, ApJ, 481, 500

De Pontieu, B., Hansteen, V. H., Rouppe van der Voort, L., van Noort, M., \& Carlsson, M. 2007a, ApJ, 655, 624

De Pontieu, B., McIntosh, S., Hansteen, V. H., et al. 2007b, PASJ, 59, 655

De Pontieu, B., Title, A. M., Lemen, J. R., et al. 2014, SoPh, 289, 2733

Dere, K. P. 2011, BAAS, 218, 134.02

Dere, K. P., Landi, E., Young, P. R., et al. 2009, A\&A, 498, 915

Dudík, J., Del Zanna, G., Dzifčáková, E., Mason, H. E., \& Golub, L. 2014, ApJL, 780, L12

Fabbian, D., \& Moreno-Insertis, F. 2015, ApJ, 802, 96

Fang, F., Manchester, W., IV, Abbett, W. P., \& van der Holst, B. 2012, ApJ, 745,37

Feldman, U. 1992, PhyS, 46, 202

Golding, T. P., Carlsson, M., \& Leenaarts, J. 2014, ApJ, 784, 30

Goodman, M. L. 2012, ApJ, 757, 188

Grevesse, N., \& Sauval, A. J. 1998, SSRv, 85, 161

Gudiksen, B. V., Carlsson, M., Hansteen, V. H., et al. 2011, A\&A, 531, A154

Gudiksen, B. V., \& Nordlund, ̊̀ 2005, ApJ, 618, 1020

Hansteen, V. 1993, ApJ, 402, 741

Hansteen, V., De Pontieu, B., Carlsson, M., et al. 2014, Sci, 346

Hansteen, V. H., De Pontieu, B., Rouppe van der Voort, L., van Noort, M., \& Carlsson, M. 2006, ApJL, 647, L73

Hansteen, V., Guerreiro, N., De Pontieu, B., \& Carlsson, M. 2015, ApJ, 811, 106

Hansteen, V. H., Hara, H., De Pontieu, B., \& Carlsson, M. 2010, ApJ, 718,1070

Hayek, W., Asplund, M., Carlsson, M., et al. 2010, A\&A, 517, A49

Heggland, L., De Pontieu, B., \& Hansteen, V. H. 2007, ApJ, 666, 1277

Joselyn, J. A., Munro, R. H., \& Holzer, T. E. 1979, ApJS, 40, 793

Judge, P., Carlsson, M., \& Wilhelm, K. 1997, ApJL, 490, L195

Leenaarts, J., Carlsson, M., Hansteen, V., \& Rutten, R. J. 2007, A\&A, 473, 625

Martínez-Sykora, J., De Pontieu, B., Hansteen, V., \& Carlsson, M. 2015a, RSPTA, 373, 40268

Martínez-Sykora, J., De Pontieu, B., Hansteen, V., \& McIntosh, S. W. 2011a, ApJ, 732, 84

Martínez-Sykora, J., De Pontieu, B., Leenaarts, J., et al. 2013, ApJ, 771, 66

Martínez-Sykora, J., Hansteen, V., \& Carlsson, M. 2008, ApJ, 679, 871

Martínez-Sykora, J., Hansteen, V., \& Carlsson, M. 2009a, ApJ, 702, 129

Martínez-Sykora, J., Hansteen, V., DePontieu, B., \& Carlsson, M. 2009b, ApJ, 701, 1569

Martínez-Sykora, J., Hansteen, V., \& Moreno-Insertis, F. 2011b, ApJ, 736, 9

Martínez-Sykora, J., Rouppe van der Voort, L., Carlsson, M., et al. 2015b, ApJ, 803,44

Nordlund, Å 1982, AAP, 107, 1

Olluri, K., Gudiksen, B. V., \& Hansteen, V. H. 2013a, AJ, 145, 72

Olluri, K., Gudiksen, B. V., \& Hansteen, V. H. 2013b, ApJ, 767, 43

Olluri, K., Gudiksen, B. V., Hansteen, V. H., \& De Pontieu, B. 2015, ApJ, 802,5

Pereira, T. M. D., Asplund, M., Collet, R., et al. 2013, A\&A, 554, A118

Sivaraman, K. R., Gupta, S. S., Livingston, W. C., et al. 2000, A\&A, 363, 279

Sivaraman, K. R., \& Livingston, W. C. 1982, SoPh, 80, 227

Skartlien, R., Stein, R. F., \& Nordlund, Å 2000, ApJ, 541, 468

Steffens, S., Deubner, F.-L., Fleck, B., \& Wilhelm, K. 1997, in Tracing CA K Grains through the Chromosphere into the Transition Region 404, ESA Special Publication, Fifth SOHO Workshop: The Corona and Solar Wind near Minimum Activity, ed. A. Wilson (Noordwijk: ESA), 685

Testa, P., De Pontieu, B., Allred, J., et al. 2014, Sci, 346

Tortosa-Andreu, A., \& Moreno-Insertis, F. 2009, A\&A, 507, 949

Wedemeyer, S., Freytag, B., Steffen, M., Ludwig, H.-G., \& Holweger, H. 2004, A\&A, 414, A1121 